\title{
NOTE PER UNA TIPOL̃OGIA DEI BALSAMARI ROMANI A FONDO PIATTO
}

\author{
POR
}

ANDREA CAMILLI

Università degli Studi di Roma «La Sapienza»

\begin{abstract}
RESUMEN
Se estudia la producción de recipientes de perfume de fondo plano, de época romana, habitualmente denominados unguentaria. A partir de agrupaciones morfológicas se establece una primera clasificación tipológica abierta. Dado que no resulta fácil definir una línea evolutiva en cuanto a formas y difusión, se concluye que estas vasijas, salvo excepciones, forman parte de una producción de difusión regional, no preparada para la exportación.
\end{abstract}

\section{SUMMARY}

This paper refers to the flat-bottomed production of Roman age ceramic vases expressly made to contain perfumes. Starting from morphological associations a first effort at an open typology is presented. Considering the typological aspect, it is not easy for the time being to define a complete line of evolution in shapes and diffusion; what is certain though is that perfume vases seem to be of local production and diffusion and, with rare exceptions, do not seem to be goods for a long distance trade.

Con il termine unguentari, o meglio balsamari, si è soliti definire quelle piccole bottiglie di ceramica, di dimensione variabile dai 4-5 ai 20-30 centimetri di altezza che, a partire dall'età ellenistica, sostituiscono le classiche lekithoi come contenitori di sostanze a base oleosa. La scelta del termine che definisce questa particolare classe ceramica non è casuale. Il termine unguentarius non è, credo, assolutamente da preferire; questo è attestato, tranne che in un singolo caso ${ }^{1}$, esclusivamente come riferito ai commercianti di sostanze profumate ${ }^{2}$, mentre l'originale nome del contenitore, che specie nella prima età imperiale varia troppo esiguamente nella forma per poter essere contraddistinto da termini differen-

\footnotetext{
1 Peraltro molto discutibile (Poggesi in Ricci 1985: 28).

2 Cfr., tra le molte, Cic. ad Att. XII.46 ... Quid minus probandum quam Plotium Unguentarium ... ; ILLRP 826/AE 1932.22 ... Epaphroditus Novi (servus) / ung $<$ u>entarius de Sa / cra Via ...; ILLRP 824/CIL X, 3975 ... C . l. Philar / gyro, un / gentario ... ; ILLRP 825/CIL IX, 471 ... L . Novius L. L. L . libert(ei) / Prepo , Dionysi[us] / unguentariei ...; CIL VI, 1974 ... M. Poblicius [-] / NIcanor. ung(uentarius) [-] / de . Sacra . via ...
}

ziati, potrebbe essere individuabile in un accenno che fornisce Plauto ${ }^{3}$ in Ampulla ${ }^{4}$.

L'assenza di una sistematizzazione tipologica dei balsamari romani è cosa piuttosto evidente ${ }^{5}$; l'unico lavoro generale, peraltro ormai obsoleto, che riguarda questa classe è il celeberrimo articolo della Forti (1962) che è tuttavia rivolto solo alle forme della prima età ellenistica e tralascia completamente $\mathrm{i}$ più tardi balsamari a fondo piatto. Un primo tentativo di definizione è stato operato dalla AndersonStojanovich $(1987)^{6}$; è tuttavia piuttosto indicativo (differenzia solo cinque tipi) e presenta una bibliografia di confronto, seppure molto ampia, fortemente caratterizzata dall'ambiente balcanico.

La scarsità dei contesti editi, la relativa difficoltà di datazione ${ }^{7}$, e la stupefacente riottosità degli editori nel pubblicare sezioni anzichè foto di queste forme, riduce considerevolmente le possibilità di tipologizzazione. Pubblicazioni di grandi ed estesi contesti sono in realtà pochissime; tutt'ora fondamentale è la pubblicazione degli scavi di Ampurias (Almagro 1955) alla quale si deve il disegno di almeno la metà dei tipi noti; altre forme sono già state edite nel già citato lavoro di Virginia Anderson (1977), purtroppo non cronologizzato; per il resto, e

${ }^{3}$ Plauto, Rudens, 756: ... ni erit tam sincerum, ut quivis dicat ampullarius loptimum esse operi faciundo corium et sincerissimum...; il fatto che ne faccia riferimento Plauto ovviamente esclude la possibilità che il termine ampulla venisse usato per i balsamari vitrei. Vedi anche CIL XII, 4455 ... C.L. Philomusus/ampullarius/ Frugi ...

${ }^{4}$ Per il termine in italiano cfr. Camilli 1995: 159

5 L'intera classe ceramica oggetto del presente lavoro, infatti, è stata a volte definita con il confronto con una unica forma, la Haltern 10 (Loeschke 1909: 201-202; in questa tipologia sotto il numero 111.1).

${ }^{6}$ Si tratta di un approfondimento di una esercitazione di laurea sulla necropoli di Stobi (Macedonia) (Anderson 1977), esercitazione che ha fornito un gran numero dei balsamari inseriti in questa tipologia. Purtroppo l'Autrice, in questo lavoro, non ha presentato, se non in pochissimi casi, alcuna datazione dei contesti.

7 La gran parte dei balsamari in ceramica infatti, si rinviene solitamente in sepolture di non altissimo livello sociale, che per la scarsità del corredo consentono difficili appigli cronologici. 


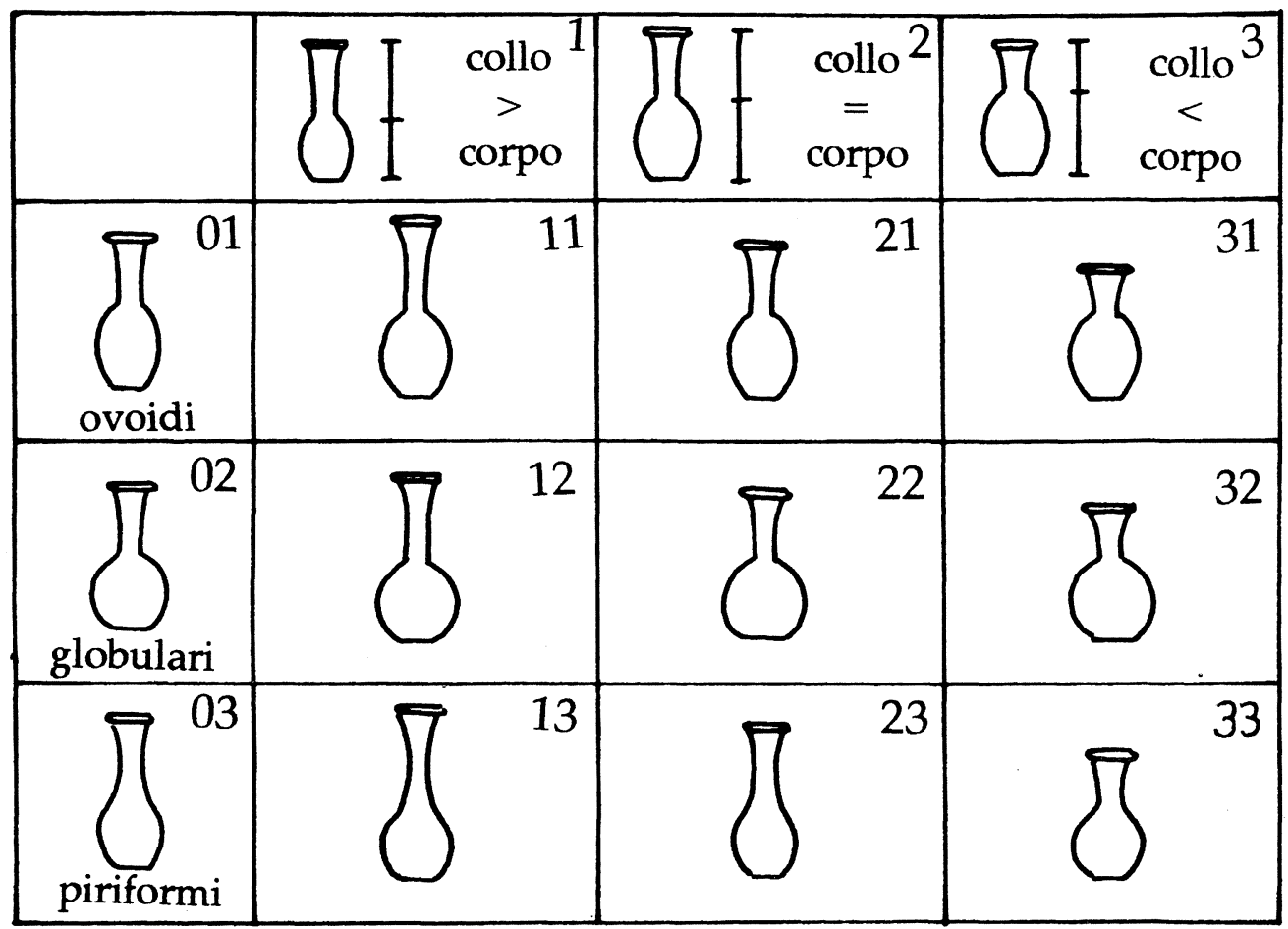

Fig. 1

particolarmente per l'Italia, dobbiamo fare affidamento per lo più a singoli contesti editi variamente e sporadicamente.

La necessità dell'impostazione di una struttura tipologica «aperta», dove cioè sia possibile inserire in qualsiasi momento nuovi tipi, in uno studio ceramologico, è stata definitivamente sancita dal Morel (1981: 33) nel suo fondamentale studio sulla ceramica a vernice nera; è innegabile che lo schema che si è cercato di utilizzare in questo primo tentativo tipologico è ispirato al suo sistema.

Una osservazione che risulta subito evidente è come nei balsamari l'analisi del solo orlo sia estremamente poco caratterizzante il tipo ${ }^{8}$; per questi il singolo ridotto frammento molto raramente riesce a dare indicazioni tipologiche se non associato con altri balsamari interi. Si è cercato di definire quindi la forma generale del tipo basandosi su due criteri paralleli: il rapporto tra l'altezza del collo e del corpo e la forma del corpo. Sono state quindi definite (fig. 1) tre sottoclassi principali, indicate con i nu-

${ }^{8}$ Cosa che non avviene, ad esempio, per la ceramica d'uso comune; piuttosto come nella ceramica a pareti sottili, dove l'identificazione delle forme e del tipo passa necessariamente per l'osservazione della forma complessiva del reperto. meri 1 (= collo allungato rispetto al corpo), 2 (= collo proporzionato al corpo), 3 (= collo corto), all'interno delle quali si è cercato di ordinare le varie serie in tre categorie formali, indicate con i numeri 01 (= corpo ovoide), 02 (= corpo globulare), 03 (= corpo piriforme). Le attestazioni raccolte sono state quindi sudivise in «serie» con analoghe caratteristiche formali, nell'ambito delle quali si differenziano i vari «tipi», cioè le varie attestazioni con identiche o di poco distinte caratteristiche. Si è ottenuta così una seriazione «aperta», nella quale sarà possibile inserire, con l'evoluzione degli studi, eventuali altre attestazioni sfuggite alla classificazione. La ricerca di un confronto potrà quindi partire dallo schema della fig. 1, per poi scendere nel dettaglio delle serie tipologiche.

Il ridottissimo spettro cronologico nel quale si è costretti ad operare (poco più di un secolo, dalla metà del I secolo a.C. alla seconda metà del I secolo d.C.) si è rivelato un problema di non piccola entità; dopo la fine della produzione della ceramica a vernice nera, il materiale di provenienza tombale difficilmente riesce ad essere datato con grande dettaglio; un arco cronologico di cinquanta anni è un risultato più che accettabile. Si è quindi cercato, qualora possibile, di ridefinire cronologicamente i contesti per poi ricollocare le varie attestazioni 


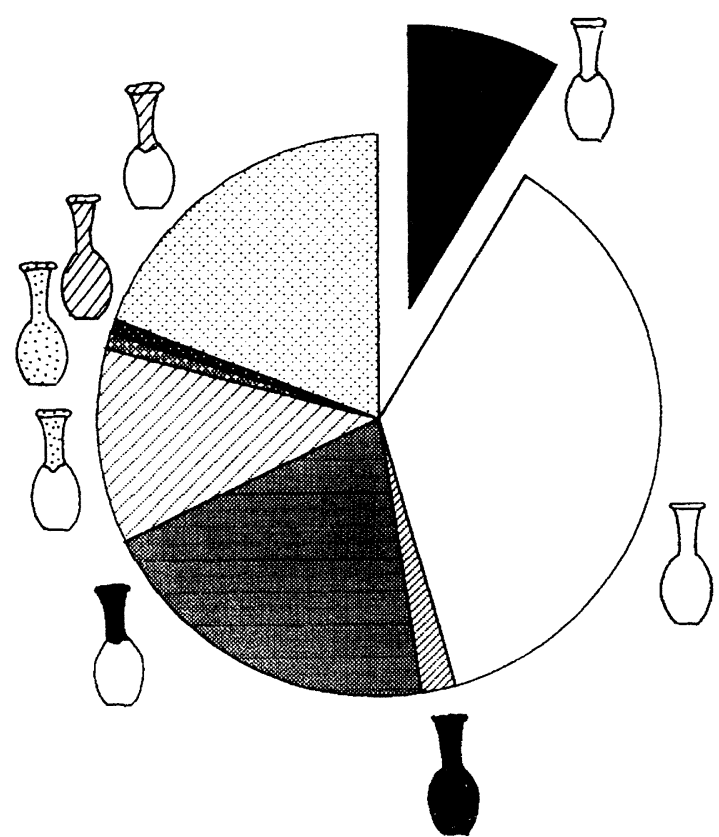

collo a vernice non identificabile o non specificata $8,89 \%$ acromi $36,67 \%$

corpo a vernice nera $1,85 \%$

collo a vernice nera $20,37 \%$

collo a vernice rossa $11,48 \%$

corpo a vernice rossa $0,74 \%$

corpo a vernice bruna $0,74 \%$

collo a vernice bruna $8,89 \%$

Fig. 2

in serie di tipo morfologico accludendo le cronologie attestate; in alcuni casi si è dovuto fare affidamento sul giudizio dell'Editore; come norma tuttavia si è preferito evitare queste datazioni non ricontrollabili.

Un punto fondamentale che è risultato da questa operazione di riordino è che i balsamari non sembrerebbero essere stati oggetto di commercio a grandi distanze. Le caratteristiche delle argille, quando è stato possibile osservarle direttamente, fanno pensare a delle produzioni di ambito piuttosto ristretto, regionale se non locale; si potrebbe pensare, al limite, a una diffusione più di forme e mode che di pezzi. I medesimi tipi infatti sono attestati, in differenti località, in vari tipi di ceramica ed ingubbiatura; ad esempio quello classificato come 131.1 è attestato a Norchia e a Corinto con il collo a vernice bruna, a Nave in depurata acroma e ad Ampurias con il collo a vernice nera, in depurata acroma e in almeno due tipi di argille diversi ${ }^{9}$.

Non risulta quindi facile, allo stato attuale della ricerca, un discorso di produzioni e attribuzioni. E' probabile che le essenze profumate venissero importate in altro genere di recipienti e quindi distribuite

${ }^{9} \mathrm{Al}$ contrario la Vegas, nel suo ormai classico studio sulle ceramiche utilitarie (1973: 153), sembra ritenere possibile una fabricación bastante centralizada en cierto número de alfares desde donde se exportaban ...; un dettagliato studio delle argille potrebbe chiarire questo punto. localmente attraverso officine collegate ai vari unguentarii ${ }^{10}$. Seppure in ambito cronologico diverso, sono stati ad esempio rinvenuti contenitori da trasporto per essenze oleose profumate in un relitto in Turchia, a Serçe Limani (Pulak - Townsend 1987: 48).

Altro argomento che potrebbe risultare di un certo interesse è la differenziazione dei vari tipi di verniciatura attestati. Sulla totalità dei balsamari considerati in questo lavoro (fig. 2) osserviamo come il $36 \%$ degli esemplari schedati risulti acromo. Se l'ingubbiatura o la verniciatura del pezzo aveva principalmente una funzione utilitaria, di impermeabilizzazione della superficie, gli esemplari acromi andrebbero quindi collocati in produzioni di bassa qualità o effettuate con argille piuttosto compatte. La verniciatura del collo, che è in realtà una verniciatura per immersione parziale del vaso soprattutto rivolta all'interno di questo, assume quindi caratteristiche puramente funzionali anche se è innegabile che possa essere stata influenzata da un certo gusto decorativo; ciò sembrerebbe dimostrato dall'estrema esiguità percentuale dei ben curati pezzi completamente verniciati.

\footnotetext{
${ }^{10}$ Come farebbe pensare l'estrema variabilità della capienza dei vasi; un tentativo di verifica della rispondenza della capienza dei balsamari ad unità di misura note in $\mathrm{Ca}$ milli 1995a: 568-571.
} 


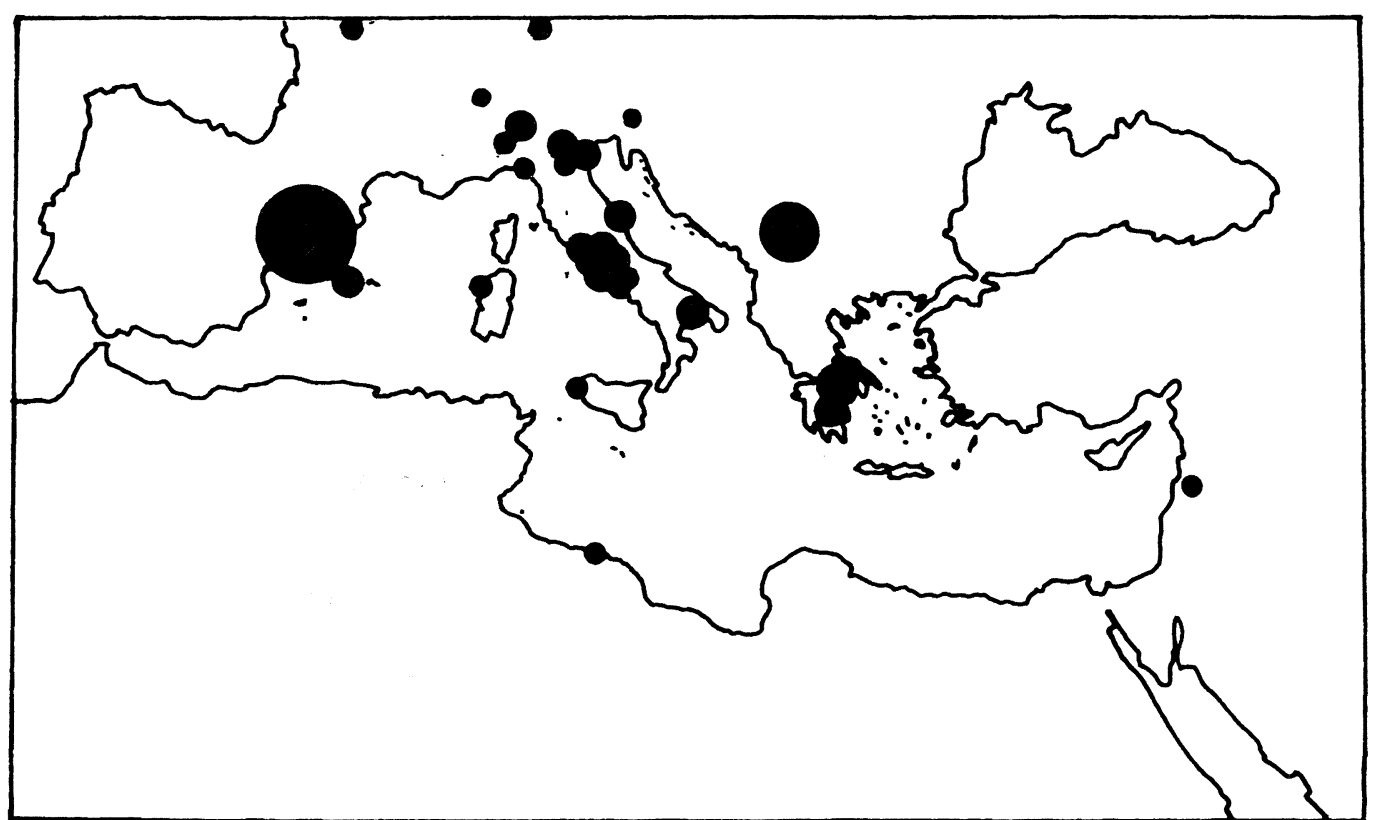

Fig. 3

Non sembra esserci stata una differenziazione cronologica e/o geografica nell'uso di vernici nere, rosse e brune. Certo è che in questa classe la caratteristica ingubbiatura della ceramica a vernice nera sembra essere stata usata molto più a lungo della classe omonima. E' tuttavia evidente come tutte queste considerazioni possano essere influenzate dalla preponderanza dei rinvenimenti di ambiente spagnolo in questo studio. Nella fig. 3 è presentato infatti un quadro schematico della distribuzione degli esemplari considerati; da notare come i materiali provenienti da Ampurias siano preponderanti.

E' comunque possibile un primo abbozzo di analisi dell'evoluzione formale di questa classe, forse da collegare con le differenti diffusioni di essenze profumate che, come per tutti gli altri beni di lusso, devono aver seguito delle mode.

Nella prima metà del I secolo a.C. le ultime produzioni di balsamari fusiformi ${ }^{11}$ tendono ad un sempre maggiore assottigliamento del piede «a chiodo» per arrivare alla completa atrofizzazione nelle nostre forme a fondo piatto; contemporaneamente si assiste ad un allungamento ed assottigliamento del collo, con orli a fascetta molto ridotti, caratteristiche queste che presuppongono la presenza di essenze piuttosto liquide e forse volatili. Con la diffusione

1 I Va e Vb secondo la classificazione della Forti (1962: 151-152). dei nuovi balsamari a fondo piatto, intorno al 50 a.C., assistiamo ad un generale aumento dell'ampiezza dei colli e ad un aumento della superficie orizzontale degli orli (ad esempio le serie 121, 231) che, ripiegati o meno, potrebbero aver assunto la stessa funzione degli orli a tesa degli aryballoi arcaici e delle lekythoi classiche. Già dai primi anni del I secolo d.C. sembra di poter assistere a due parallele evoluzioni formali; da un lato, la sempre crescente diffusione del vasellame di vetro, che progressivamente soppianterà $\mathrm{i}$ balsamari in ceramica, porta a diverse «serie» di imitazioni di forme vitree, quali le $232,233,234,235^{12}$; contemporaneamente, probabilmente per un generale aumento della densità dei contenuti, si assiste a un progressivo strombarsi dei colli e ad una atrofizzazione degli orli (vedi le serie 334 e 335 ), caratteristica questa che sembra accompagnare tutte le forme più tarde di balsamari, fino alla fine della produzione ${ }^{13}$.

12 Tutte evidentemente derivate dalla forma Isings 28a (Isings 1957).

13 Devo ringraziare, per l'aiuto ed incoraggiamento fornitomi, G. Alvino; un ringraziamento particolare per i preziosi suggerimenti fornitimi nel corso del lavoro va a M. Incitti; un grazie per avere sopportato milioni di ritagli e intemperanze ad A. De Laurenzi. 


\section{TIPOLOGÍA}

Nella stesura della tipologia sono state utilizzate le seguenti abbreviazioni:
p. = pasta
$\mathrm{v} .=$ vernice
alt. = altezza
n. dat. = non databile
n. des. = non descritto dall'Editore.

\section{SERIE 111}

Corpo ovoide, collo allungato tronco-conico o cilindrico leggermente strombato, orlo più o meno estroflesso, a fascetta arrotondata sul punto di massima espansione, fondo piatto più o meno ampio.

Il tipo 111.1 è il balsamario piriforme in assoluto più diffuso, ed ha uno spettro cronologico piuttosto ampio, con un addensamento di attestazioni nel primo trentennio del I secolo d.C.; le forme successive, di produzione verosimilmente ispanica, vanno collocate in età tiberiano-claudia.

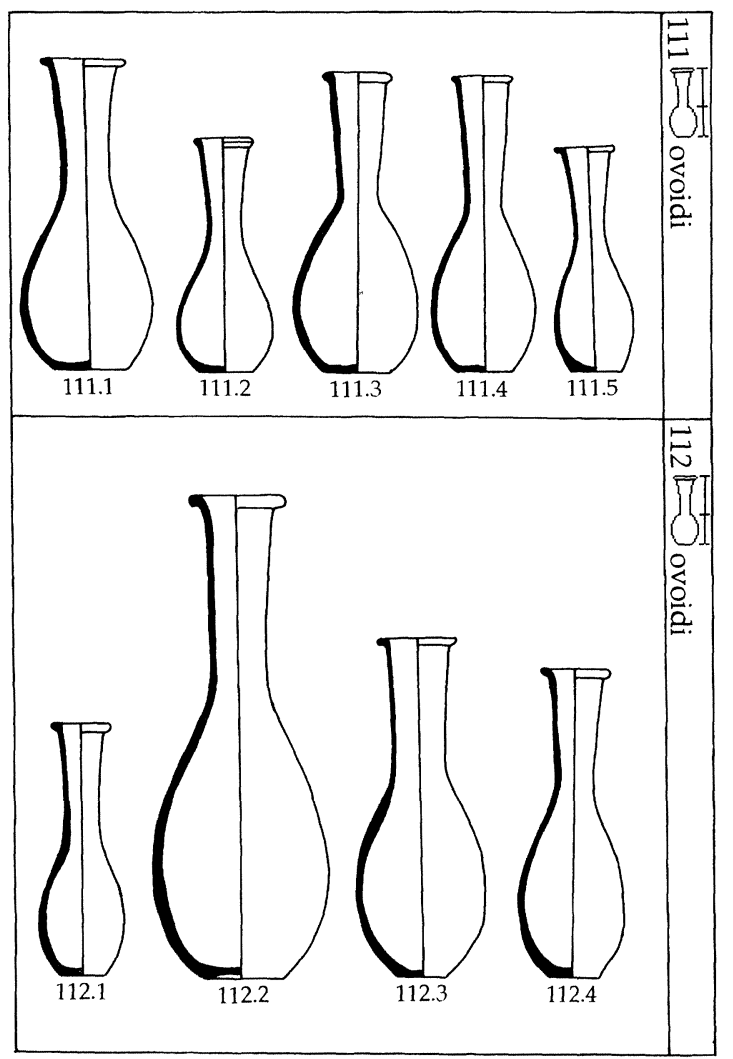

Fig. 4
Tipo 111.1

Castel D'Asso (Colonna 1970: 190, tav. CCCCXI, t.ba 17 Dromos n. 6: 190), p. rossa, collo a v. rossa, alt. 13,5, non stratificato ma associato con ceramica a pareti sottili del 15-30 d.C.;

Corinto (Blegen - Palmer - Young 1964: 167, 295 , pl. 76, 505-4; 167, 295, pl. 76, 505-3), in due esemplari, p. arancio, collo verniciato, alt. 9 e 12,8, gruppo augusteo «ii»;

Segusio (TO) (Brecciaroli Taborelli 1990: 109 nr. 79), uguale al precedente, frammentario, p. bruno chiaro/giallo rossiccio, v. bruna metallescente, fase A1 (augusteo-tiberiana, forse più tiberiana);

Gropello Cairoli (PV) (Fortunati Zuccala 1979: 46-7: 53), tre esemplari a v. bruna su collo, altezze $8,5,9,8$, prima metà del I secolo d.C.;

Haltern (Loeschke 1909, pl. XXI, 10: 201-2), scala bastarda, n. des., n. dat.;

Padova (Zampieri 1991: 172, ingr. 233529, inv. 180(?): 189), alt. 8,9, n. des., n. dat.;

Roma (Felletti Maj 1957: 332 fig. 1), in 2 esemplari, p. giallo-rosa, v. rossa su collo, altezze 10 e 9,5, non databili;

Lucus Feroniae (inedito), p. rosa, v. rossa su collo e interno, contesto presumibilmente augusteo;

Ampurias (Almagro 1955: 58 n. 9), collo a v. rosso-nerastra, alt. 9,2, associato con moneta del 42 a.C.

\section{Tipo 111.2}

Pollentia (Arribas 1983: 323, 343, nr. 54), p. beige chiara, collo e interno a ingubbiatura castana, alt. 9,9 , n. dat.

\section{Tipo 111.3}

Ampurias (Almagro 1955: 83 n. 4; 84 n. 3; 84 n. 5; 70 n. $5 ; 70$ n. $4 ; 71$ n. 4), in sei esemplari; p. scura, alt. 12,4, età tiberiana; con collo più allungato, p. chiara, alt. 14,3 , n. dat.; p. chiara, alt. 9,4 , n. dat.; p. rossastra, alt. 10,4, n. dat.; p. rossastra, alt. 10,5, n. dat.; leggermente deformato, p. grigio scuro, collo a v. nera, alt. 9 , età tiberiana; con orlo sporgente, quasi a tesa, p. rossastra, collo a v. nera, alt. 13,2, n. dat.

\section{Tipo 111.4}

Ampurias (Almagro 1955: 183 n. 11; 183 n. 10; 229 n. 11), in tre esemplari; p. scura, alt. 12,3, età tiberiano-claudia; n. des., alt. 11,8, età tiberianoclaudia; n. des., alt. 9,6, n. dat.

\section{Tipo 111.5}

Ampurias (Almagro 1955: 229 n. 10), n. des., alt. 9,3, n. dat. 


\section{SERIE 112}

Corpo ovoide, collo allungato cilindrico, talvolta leggermente strombato, orlo ripiegato più o meno arrotondato, talvolta a tesa, appiattito superiormente, fondo piatto di piccole dimensioni, più o meno distinto.

I tipi originari sono il 112.3 ed il 112.7 , da porre nell'ultimo trentennio del I secolo d.C.; la serie si evolve con i 112.1, 112.2, 112.5, 112.6 in età augustea per produrre i 112.4 e 112.8 in età tiberiana.

\section{Tipo 112.1}

Ampurias (Almagro 1955: 112 n. 2; 112 n. 1; 105 n. $6 ; 188$ n. 22), in quattro esemplari; con resti di pittura, alt. 10,6, n. dat.; con resti di pittura, alt. 10 , n. dat.; con resti di pittura, alt. 11,7, augusteo; con il collo leggermente più allungato, alt. 8,4, p. scura, età tiberiana;

Musarna (Emiliozzi 1974: 50, tav. XVII,8, tav. $\mathrm{XXX}, 8)$, p. rosso arancio, collo a v. rossa, alt. 12,7, contesto del 20-30 d.C.;

Corinto (Blegen - Palmer - Young 1964: 167, 297, pl. 77, 510-2; 167, 295, pl. 76, 506-4), in due esemplari; altezze 10,2-10,8, p. arancio, collo sopradipinto (in bruno?), «group ii» augusteo.

\section{Tipo 112.2}

Stobi (Anderson 1977: 259, fig. 196 n. 757), collo a v. bruno-rossiccia, senza cronologia;

Corinto (Blegen - Palmer - Young 1964: 167, 297, pl. 77 , 510-3; 167, 295, pl. 76, 505-2; 167 , 295 , pl. 76, 506-2), in tre esemplari, p. arancio, collo dipinto (in bruno?), altezze 16,3, 16,6, 15,8, «group ii» augusteo.

\section{Tipo 112.3}

Stobi (Anderson 1977: 260, fig. 199, n. 765), v. rosso-bruna sul collo, n. dat.;

Nave (Passi Pitcher 1987: 37-38), p. marrone scura, alt. 10, contesto compreso tra il 30 a.C. e lo O;

Salonicco (Hayes 1976: 34; 105 n. 161), alt. 12,3 , p. arancio-rossiccia, sabbiosa, fiammata di grigio, acromo, fuori contesto, apparentemente, per l'Editore, di produzione macedone.

\section{Tipo 112.4}

Pollentia (Arribas 1983: 323, 343, n. 55), p. beige chiara, ingubbiatura castana diluita su tutto il corpo, più scura su collo e internamente, alt. 12,8, contesto della prima metà del I secolo d.C.;

Ampurias (Almagro 1955: 229 n. 9; 188 n. 20; 188 n. 21 ; 105 n. 7), in quattro esemplari; n. des., alt. 10, n. dat.; con orlo meno accentuato, p. chiara, alt. 9,3, età tiberiana; uguale al precedente, p. chiara, alt. 11,3, età tiberiana; con resti di v., alt. 11,2, augusteo.

\section{Tipo 112.5}

Lucus Feroniae (inedito), p. grigia, v. bruno grigiastra su collo e internamente, contesto presumibilmente augusteo.

\section{Tipo 112.6}

Lucus Feroniae (inedito), p. arancio, v. bruna su collo e internamente, contesto presumibilmente augusteo.

\section{Tipo 112.7}

Ampurias (Almagro 1955: $57 \mathrm{nn} .10$ e 14; $58 \mathrm{nn}$. 6, 7, 8; 229 nn. $1,2,6,9 ; 81$ n. $2 ; 81$ n. $3 ; 83$ n. 6 ; 75 n. $6 ; 85$ n. 1) in quattordici esemplari; due esemplari, alt. 10 ca., p. grigia, collo a v. nera, fine del I secolo a.C.; tre esemplari con collo più tozzo, alt. $9,9,10,10,2$ ca., collo a v. rosso-nera, associati con moneta del 42 a.C.; quattro esemplari con orlo leggermente ripiegato e arrotondato, n. des., n. dat., alt.

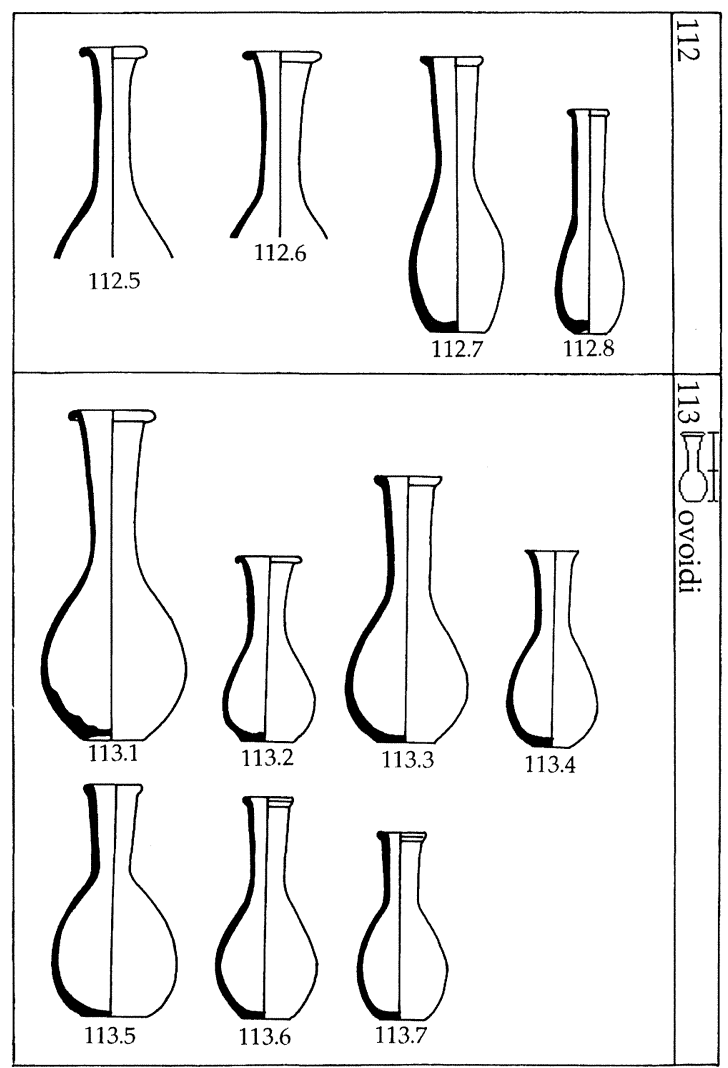

Fig. 5 
12,5, 11,8, 10,6, 10,3; p. scura, alt. 12,3, n. dat.; p. scura, alt. 12,2, n. dat.; p. scura, alt. 10,4, età tiberiana; con collo a v. rossa, alt. $9,5, \mathrm{n}$. dat.; con collo a v. rossa, alt. $10,3, \mathrm{n}$. dat.

\section{Tipo 112.8}

Ampurias (Almagro 1955: 188 n. 19), p. chiara, alt. 9,5, età tiberiana.

\section{SERIE 113}

Corpo ovoide piuttosto rigonfio, collo più o meno allungato, troncoconico, orlo estroflesso ripiegato e arrotondato, talvolta assottigliato o arrotondato, piccolo fondo piatto ben distinto.

I due tipi più antichi sono il 113.2 e 113.3 (dall'ultimo trentennio del I secolo a.C.), che generano le successive varianti di età augustea (113.1) e giulio-claudia (113.3-4). Gli ultimi due tipi, non databili, sono probabilmente da ascrivere a questa fase. Il tipo 113.5 ha rapporti con il 334.7.

\section{Tipo 113.1}

Stobi (Anderson 1977: 260, fig. 196 n. 762), collo a v. bruno-rossiccia, senza cronologia;

Corinto (Blegen - Palmer - Young 1964: 167, 299, pl. 78, 520-1), p. bruno-rossastra scura, pareti spesse, alt. 9,4, augusteo (group ii).

\section{Tipo 113.2}

Argo (Bruneau 1970: 481, 483, 517, n. 61.15), p. beige, alt. 7,8 , contesto della seconda metà del $\mathrm{I}$ secolo a.C.

\section{Tipo 113.3}

Ampurias (Almagro 1955: 49 n. 4; 49 n. 8; 57 n. 11; 57 n. 11; 92 n. 3; 68 n. 1), in sei esemplari; p. chiara, alt. 10, ultimi decenni del I secolo a.C.; frammentario, ma con orlo a fascia solcato da una linea sottilissima, collo a v. nera, ultimi decenni del I secolo a.C.; con orlo leggermente pendente, p. grigia, collo a v. nera, ultimi decenni del I secolo a.C.; identico, dallo stesso contesto, frammentario; con tracce di v. sul collo, alt. 9,8, età tiberiano-claudia; p. rossastra, acromo, alt. 11, augusteo.

\section{Tipo 113.4}

Ampurias (Almagro 1955: 196 n. 21; 252 n. 21; 92 n. 4), in tre esemplari; n. des., alt. 8, età tiberiana; con orlo leggermente più ingrossato ed appiattito, n. des., alt. 9,4, età augusteo-tiberiana; con orlo e pareti del collo più ingrossate, tracce di v. sul collo, alt. 9,6, età tiberiano-claudia.
Tipo 113.5

Ampurias (Almagro 1955: 229 n. 12; 252 n. 22), in due esemplari; n. des., n. dat., alt. 9,6; n. des., alt. 8,1 , età augusteo-tiberiana.

Tipo 113.6

Ampurias (Almagro 1955: 109 n. 11), n. des., n. dat., alt. 9.

Tipo 113.7

Ampurias (Almagro 1955: 109 n. 12), n. des., n. dat., alt. 7,8 .

\section{SERIE 114}

Corpo ovoide piuttosto rigonfio, collo allungato a tromba, orlo a piccola tesa arrotondata esternamente, fondo piatto, ampio e ben distinto.

Serie di età augusteo-tiberiana, con ridotto spettro cronologico.

\section{Tipo 114.1}

Lucus Feroniae (inedito), p. arancio, v. rossobruna su collo e internamente, patina bruna all'interno, contesto presumibilmente augusteo.

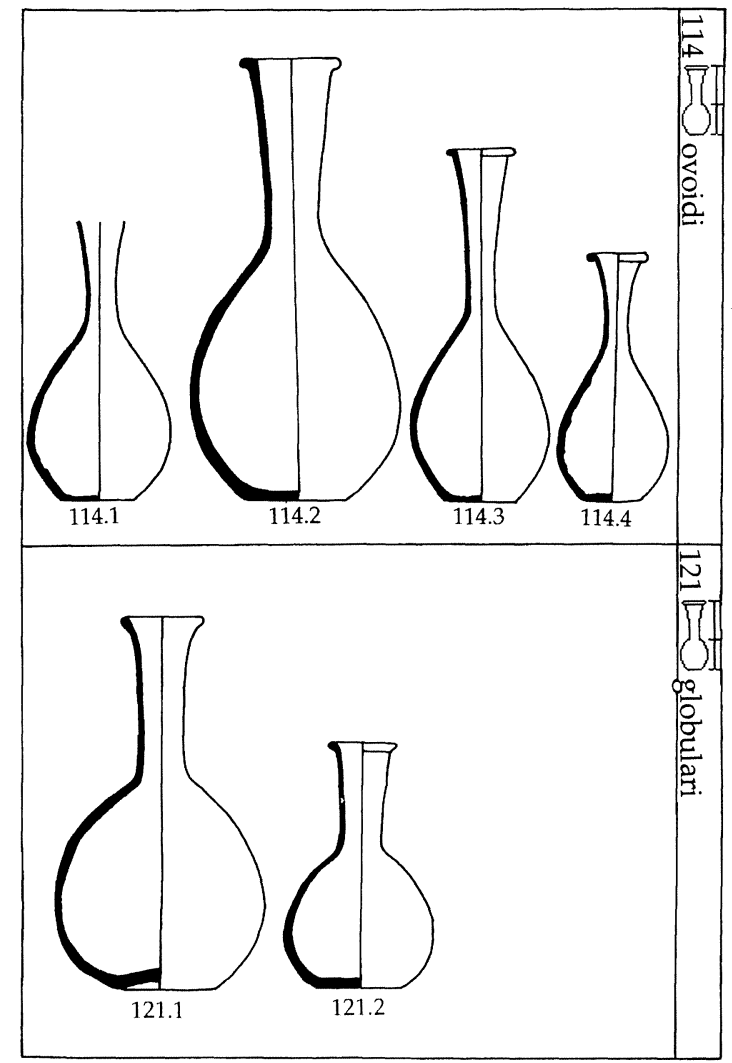

Fig. 6 
Tipo 114.2

Ampurias (Almagro 1955: 50 n. 7), v. nera, alt. 17 , n. dat.

\section{Tipo 114.3}

Ampurias (Almagro 1955: 174 n. 6), p. chiara, collo con tracce di v., alt. 14,7, età tiberiana.

\section{Tipo 114.4}

Via Amerina (inedito), p. nocciola, v. rosso-bruna sul collo, alt. 10,2, contesto augusteo.

\section{SERIE 121}

Corpo globulare, collo allungato cilindrico più o meno strombato verso l'alto, orlo estroflesso indistinto o leggermente ingrossato, fondo piatto più $o$ meno distinto.

Il tipo 121.1 è da collocare intorno alla metà del I secolo d.C.; il 121.2 è sicuramente il suo sviluppo successivo, di età giulio-claudia, anche se per il momento c'è soluzione di continuità tra le attestazioni.

\section{Tipo 121.1}

Tarquinia (Bonghi Jovino 1986: 321, fig. 324 n. $803)$, p. grigia con fiammature rosa e arancio, interno a v. nera, alt. 15,6 , contesto databile intorno al 60-30 a.C.;

Gropello Cairoli (PV) (Fortunati Zuccala 1979: 53), incompleto, p. rosa arancio, contesto degli inizi del I secolo d.C.

\section{Tipo 121.2}

Ampurias (Almagro 1955: 183 n. 8; 204 n. 6; 183 n. $9 ; 204$ n. $5 ; 183$ n. 7), in cinque esemplari; p. chiara, alt. 10 , età tiberiano-claudia; n. des., alt. 11,8 , età tiberiana; p. chiara, alt. 10 , età tiberianoclaudia; con orlo appiattito superiormente, n. des., alt. 12,6 , età tiberiana; uguale al precedente, p. chiara, alt. 10,4, età tiberiano-claudia.

\section{SERIE 122}

Corpo globulare, collo allungato cilindrico o leggermente troncoconico, orlo a fascetta arrotondata tendente a piccola tesa, fondo piatto più o meno ridotto e più o meno distinto.

Si tratta di uno spettro piuttosto ampio di varianti della stessa forma base (122.1/122.4), che deriva direttamente dalla 111 ; da porsi comunque nella prima metà del I secolo d.C.

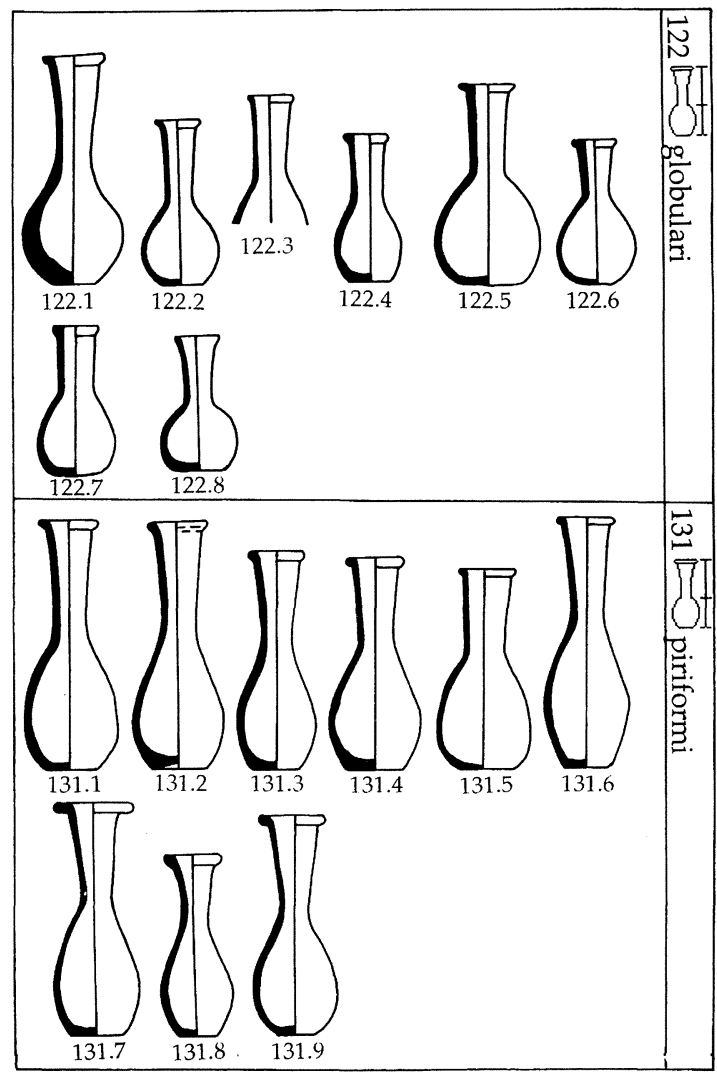

Fig. 7

\section{Tipo 122.1}

Tuscania (Moretti 1983: 146 n. 448 (tipo 96), fig. 30,74$)$, p. arancio, v. arancio sul collo, orlo e interno, alt. 9,2, augusteo.

\section{Tipo 122.2}

Pollentia (Arribas 1983: 321, 342 n. 39), p. dura e compatta color ocra chiaro, ingubbiatura dello stesso colore, alt. 7,1 , contesto della metà del I secolo d.C.;

Ampurias (Almagro 1955: 86 n. 1), di fattura più rozza e con spalla accentuata, p. rossastra, collo a $\mathrm{v}$. rossa, alt. 6,5 , n. dat.

\section{Tipo 122.3}

Padova (Zampieri 1991: 173; 191 n. 200), p. arancio rosata, fuori contesto.

\section{Tipo 122.4}

Ampurias (Almagro 1955: 57 n. 18), p. grigia, v. nera sul collo, alt. circa 6,3, ultimi decenni del I secolo a.C. 


\section{Tipo 122.5}

Ampurias (Almagro 1955: 185 n. 6; 185 n. 9), in due esemplari; p. scura, collo a v. nera, età tiberiano-claudia, alt. 8,4; p. chiara, età tiberiano-claudia, alt. 8 .

\section{Tipo 122.6}

Ampurias (Almagro 1955: 258 n. 18; 185 n. 7; 68 n. $1 ; 285$ n. $17 ; 275$ n. 69), in cinque esemplari; p. grigia, n. dat., alt. 6; p. chiara, alt. 7,2, età tiberiano-claudia; con spigolo interno dell'orlo più smussato, p. grigia, collo a v. nera, alt. 7,5, augusteo; p. grigia, alt. 5,5; v. rossa sul collo, alt. 5,9, n. dat.; con sottile cordone all'attaccatura del collo, n. des., alt. 8 , primo venticinquennio del I secolo d.C.

\section{Tipo 122.7}

Ampurias (Almagro 1955: 285 n. 15; 285 n. 16; 182 n. $1 ; 174$ n. $5 ; 285$ n. 19), in cinque esemplari; p. grigia, alt. 6 , n. dat.; p. grigia, alt. 6 , n. dat.; con orlo ripiegato e arrotondato, p. chiara, collo a v. scura, alt. 11,9 , dallo 0 all'età tiberiana; analogo al precedente, p. chiara, collo verniciato, età tiberiana; $\mathrm{p}$. grigia, alt. 6,2, n. dat.

\section{Tipo 122.8}

Ampurias (Almagro 1955: 194 n. 10), p. scura, alt. 5,7, età tiberiano-claudia.

\section{SERIE 131}

Corpo piriforme, collo allungato più o meno troncoconico, orlo estroflesso a fascetta più o meno sagomata tendente a piccola tesa arrotondata esternamente, fondo piccolo generalmente ben distinto.

E' una serie dallo spettro di attestazione piuttosto ampio, dagli ultimi decenni del I secolo a.C. alla metà del I secolo d.C.; gli ultimi tipi sono di età claudio-neroniana, come il 131.8, al quale vanno probabilmente collegati i 131.7 e 131.9; il tipo 131.7 ha rapporti con il 135.7 .

\section{Tipo 131.1}

Norchia (Colonna 1978: tav.CCCCLII, 23), alt. 10, p. camoscio-rosato, collo a v. bruna, n. dat.;

Corinto (Blegen - Palmer - Young 1964: 167 , 295, pl. 76, 506-3), p. arancio, collo a v. bruna, alt. 11, augusteo (group ii);

Sub Ascia (Passi Pitcher 1987: 37-38), p. rossastra, alt. 10,7, contesto dell'ultimo trentennio del I secolo a.C.;
Italia non meglio precisata (Hayes 1976: 33, 104 n. 156), alt. 10,4 , p. arancio-bruna, ingubbiatura arancio-marrone su collo;

Ampurias (Almagro 1955: 57 n. 13; 71 n. 5; 227 n. $4 ; 85$ n. 1), in quattro esemplari; collo a v. nera, alt. circa 10, contesto della fine del I secolo a.C.; p. grigioscura, collo a v. nera, alt. 9,3, dallo 0 all'età tiberiana; con collo meno distinto, n. des., alt. 10, n. dat.; con collo leggermente più allungato e corpo leggermente più compresso, p. chiara, alt. $9, \mathrm{n}$. dat.

\section{Tipo 131.2}

Castel D'Asso (Colonna 1970, tav. CCCCXIV, t. 36 sottofacciata n. 1,218), alt. 10,5, pasta giallo-rosata, collo a v. rossa, non stratificato;

Ampurias (Almagro 1955: 112 n. 3; 276 n. 3), in due esemplari; con tracce di v., alt. 10,3, n. dat.; con tracce di v. rossa, alt. 9,6, n. dat.

\section{Tipo 131.3}

Norchia (Colonna 1978, tav.CCCLII, 22), p. rosa, v. rossa sul collo e gran parte del corpo, alt. 8,5 .

\section{Tipo 131.4}

Ampurias (Almagro 1955: 57 nn. 15-16; 75 n. 9; 75 n. 5; 83 n. 5), in cinque esemplari; p. grigia, collo a v. nera, alt. circa 9, ultimi decenni del I secolo a.C.; leggermente più panciuto, alt. circa 9 , stesso contesto; con orlo lisciato esternamente, collo a v. rossa, alt. 6,8, n. dat.; collo a v. rossa, alt. 9,5, n. dat.; p. scura, alt. 9,7, età tiberiana.

\section{Tipo 131.5}

Ampurias (Almagro 1955: 182 n. 6; 181 n. 12; 182 n. 8), in tre esemplari; p. scura, collo a v. nera, alt. 8,2, dallo 0 all'età tiberiana; $\mathrm{p}$. scura, collo a v . arancio scuro, alt. 8, età tiberiano-claudia; p. scura, collo a v. nera, alt. 7,3, dallo 0 all'età tiberiana.

\section{Tipo 131.6}

Ampurias (Almagro 1955: 174 n. 3; 81 n. 4; 84 n. $4 ; 75$ n. 7), in quattro esemplari; p. rossiccia, collo verniciato, età tiberiana; con corpo più arrotondato, p. scura, n. dat.; p. chiara, alt. 10,5, n. dat.; con fondo meno distinto, collo a v. rossa, alt. 9 , n. dat.

\section{Tipo 131.7}

Ampurias (Almagro 1955: 70 n. 7), p. rossastra, alt. 9,7, n. dat.

\section{Tipo 131.8}

Ampurias (Almagro 1955: 84 n. 7; 88 n. 3; 68 n. $5 ; 82$ n. 1; 68 n. 4), in cinque esemplari; p. chiara, alt. 
7,3, n. dat.; leggermente più panciuto, p. grigia, alt. 7,6, n. dat.; p. rossastra, collo a v. nera, alt. 7 , età claudio-neroniana; p. chiara, alt. 9, n. dat.; p. rossastra, collo a v. nera, alt. 8,6, età claudio-neroniana.

\section{Tipo 131.9}

Ampurias (Almagro 1955: 75 n. 8; 71 n. 5; $75 \mathrm{n}$. 4), in tre esemplari; collo a v. rossa, alt. 9, n. dat.; p. scura, collo dipinto, alt. 9,8 , n. dat.; p. rossastra, collo a v. rossa, alt. 11,5 , n. dat.

\section{SERIE 132}

Corpo piriforme con spalla marcatamente convessa, collo allungato, tendenzialmente cilindrico più $\mathrm{o}$ meno strombato, orlo estroflesso più $\mathrm{o}$ meno ripiegato o ingrossato, talvolta tendente a fascetta, fondo più o meno ampio, piuttosto distinto.

Serie di difficile collocazione cronologica; il tipo 132.4 è probabilmente il più antico, così come il 132.6 e il 132.9 potrebbero essere evoluzioni della 131.5-6. Il 131.7 è probabilmente la variante recenziore del 131.6, che ha perso la definizione dell'orlo, assottigliandolo.

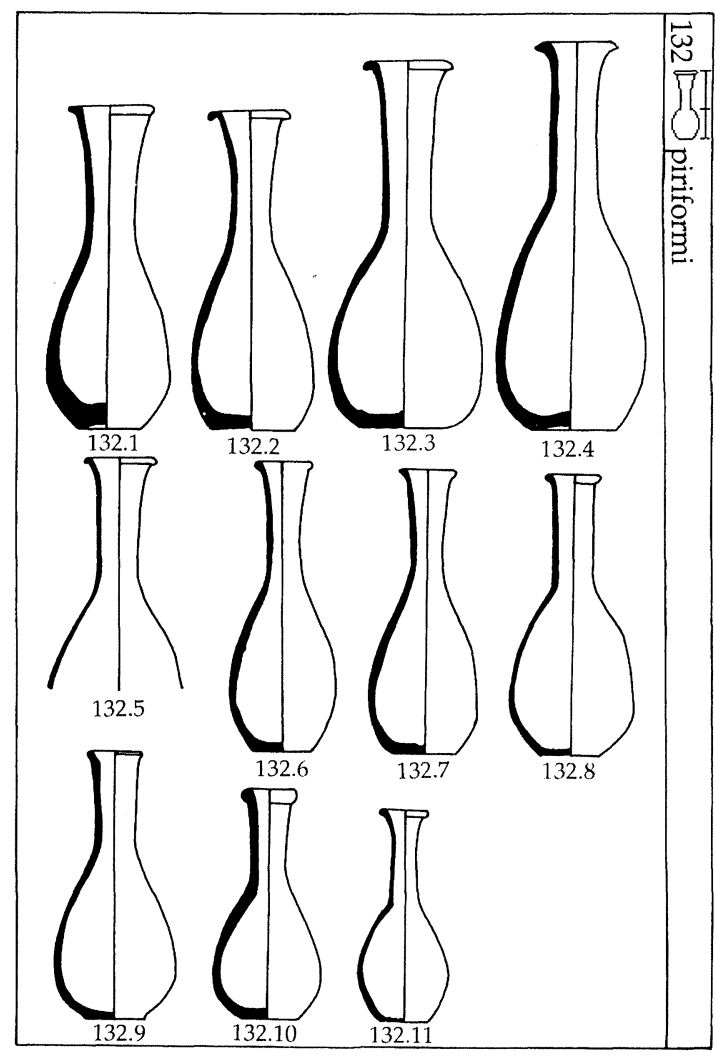

Fig. 8
Tipo 132.1

Stobi (Anderson 1977: 260, fig. 199 n. 766), v. rosso-bruna sul collo, senza datazione.

Tipo 132.2

Stobi (Anderson 1977: 260, fig. 196 n. 764), v. rosso-bruna sul collo, senza datazione.

Tipo 132.3

Stobi (Anderson 1977: 260, fig. 196 n. 761), v. rosso-bruna sul collo, senza datazione.

\section{Tipo 132.4}

Stobi (Anderson 1977: 260, fig. 196 n. 763), v. rosso-bruna sul collo, senza datazione.

Taranto (Lippolis, in De Juliis 1984: 515), v. nera opaca diluita su orlo e collo, alt. 17,5.

\section{Tipo 132.5}

Lucus Feroniae (inedito), p. rosa, v. rosso-bruna sul collo e internamente, contesto presumibilmente augusteo.

Tipo 132.6

Ampurias (Almagro 1955: 57 n. 9), p. grigia, collo a v. nera, ultimi decenni del I secolo a.C.

\section{Tipo 132.7}

Ampurias (Almagro 1955: $195 \mathrm{nn} .6-7-4)$, in tre esemplari non descritti, altezze $12,11,9,12$, n. dat

Tipo 132.8

Ampurias (Almagro 1955: 71 n. 3), p. rossastra, collo a v. nera, alt. $11,7, \mathrm{n}$. dat.

\section{Tipo 132.9}

Ampurias (Almagro 1955: 71 n. 6), p. rossastra, collo a v. nera, $n$. dat.

Tipo 132.10

Ampurias (Almagro 1955: 202 n. 1), n. des., alt. 11,4 , n. dat.

\section{Tipo 132.11}

Ampurias (Almagro 1955: 112 n. 1), p. grigia, alt. 9,8, n. dat.

\section{SERIE 133}

Corpo piriforme, largo collo allungato cilindrico o leggermente troncoconico, orlo estroflesso arrotondato, talvolta ripiegato, fondo tendenzialmente piccolo piuttosto distinto. 


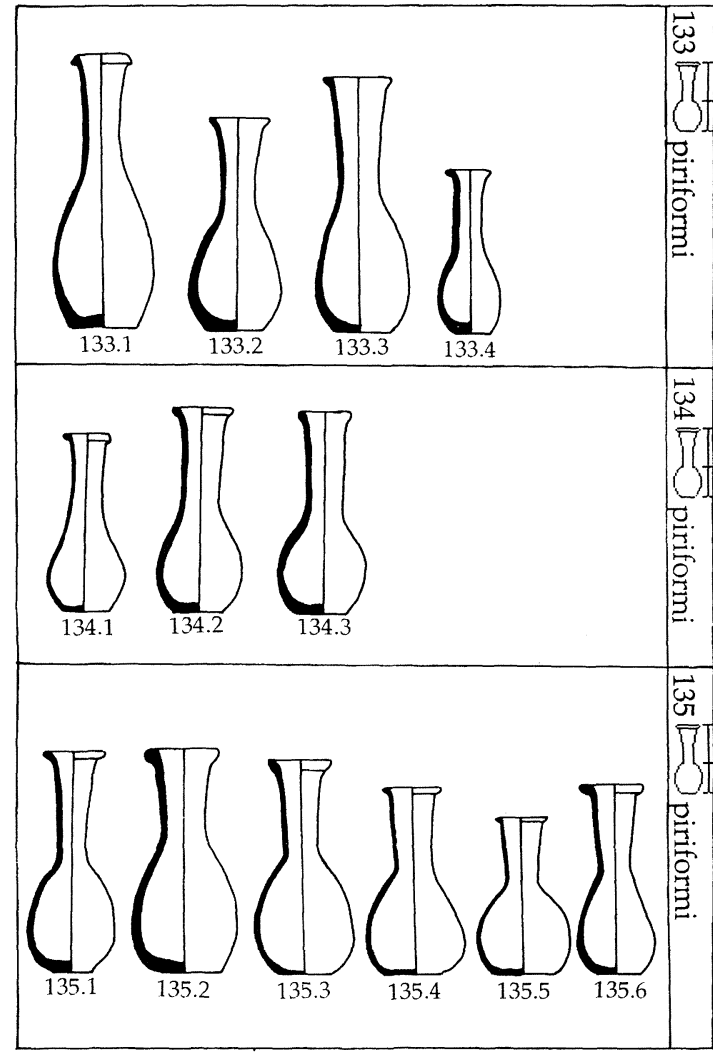

Fig. 9

Il prototipo della serie, il 133.1, è da porsi probabilmente tra la fine del I secolo a.C. e la piena età augustea, date le notevoli somiglianze con il tipo 132.4. Le restanti forme sono tutte probabilmente coeve alla 133.2 (età tiberiano-claudia).

Tipo 133.1

Stobi (Anderson 1977: 260, fig. 199 n. 768), v. rosso-bruna sul collo, n. dat.

\section{Tipo 133.2}

Ampurias (Almagro 1955: 239 n. 7; 194 n. 8), in due esemplari; tracce di pittura su collo, alt. 8,8, non dat.; p. scura, alt. 8, età tiberiano-claudia.

Leptis Magna (Joly et al. 1992: 175-176 figg. 142-143), p. arancio chiaro, v. rossa su collo, alt. 9,8 , fuori contesto.

Tipo 133.3

Ampurias (Almagro 1955: 229 n. 5, n. 4), in due esemplari; n. des., n. dat., altezze 10,6-10,7.

\section{Tipo 133.4}

Ampurias (Almagro 1955: 180 n. 3), p. rossiccia, alt. $6,7, \mathrm{n}$. dat.

\section{SERIE 134}

Corpo piriforme compresso, largo collo allungato cilindrico, orlo estroflesso e arrotondato tendente a fascetta, fondo piccolo non sempre ben distinto.

Serie piuttosto antica, da porre nella seconda metà del I secolo a.C.

Tipo 134.1

Veio (Torelli - Pohl 1973: figg. 45, 61: 86), p. nocciola, doppia ingubbiatura, l'inferiore di colore arancio-bruno, la superiore grigio chiaro con riflessi metallici, alt. 7,5 , n. dat.

\section{Tipo 134.2}

Tarquinia (Bonghi Jovino 1986: 321, fig. 324 n. 802), p. rosa, bocca e parte del corpo a v. nero-rossastra, alt. 8,7 , in contesto databile tra il 60 e il 30 d.C.

Padova (provenienza non certa; Zampieri 1991: 172 ingr. 233526 inv. 169(?) p.189) alt. 9,9, n. des., n. dat.

Ventimiglia (Hayes 1976: 12, tav. 5 n. 47), p. beige chiara, v. bruna su collo, alt. 7,4 , databile per l'Editore tra la fine del I secolo a.C. agli inizi del I secolo d.C.

Tipo 134.3

Ampurias (Almagro 1955: 57 n. 17), p. grigia, v. nera sul collo, alt. 8,4 ca., contesto della fine del I secolo a.C.

\section{SERIE 135}

Corpo piriforme con spalla marcatamente convessa, talvolta con brusco cambio di curvatura, ampio collo allungato strombato o troncoconico, orlo estroflesso e ingrossato, tendente a tesa o fascetta ma ben marcato, fondo poco distinto.

I tipi più antichi della serie sono il 135.5 e il 135.2, dai quali derivano gli altri tipi, tramite la progressiva svasatura dell'orlo e il rigonfiamento della spalla; i tipi 135.4-5 hanno forti rapporti di similitudine con il 122.5 .

\section{Tipo 135.1}

Nave (Passi Pitcher 1987: 36-37: 179, 4), p. arancio, acromo, alt. 9,4, contesto augusteo;

Ampurias (Almagro 1955: 70 n. 6), p. rossastra, alt. 10 , n. dat.

\section{Tipo 135.2}

Ampurias (Almagro 1955: 57 n. 19), p. grigia, v. nera sul collo, alt. 9,4, ultimi decenni del I secolo a.C. 


\section{Tipo 135.3}

Ampurias (Almagro 1955: 66-67 n. 3), p. rossiccia, collo verniciato, alt. 8,5 , n. dat.

\section{Tipo 135.4}

Ampurias (Almagro 1955: 185 n. 6; 182 n. 7; 181 n. $13 ; 182$ n. 4), in quattro esemplari; p. scura, collo a v. arancio scuro, alt. 7,7, età tiberiano-claudia; p. scura, collo a v. nera, alt. 7,8, dallo 0 all'età tiberiana; p. chiara, tracce di pittura sul collo, alt. 6,1 , età tiberiano-claudia; p. scura, alt. 7,7 , dallo 0 all'età tiberiana.

\section{Tipo 135.5}

Ampurias (Almagro 1955: 87 n. 5: 71 n. 9), in due esemplari; p. scura, alt. 6,6, I secolo a.C.; con spalla più marcata, p. rossastra, collo a v. nera, alt. 5,4, n. dat.

Tipo 135.6

Ampurias (Almagro 1955: 84 n. 8), p. chiara, alt. 8, n. dat.

\section{SERIE 136}

Corpo piriforme, collo allungato cilindrico leggermente svasato, orlo a tesa tendente alla sezione triangolare, fondo ampio poco distinto.

Serie molto limitata; i due esemplari schedati hanno cronologia probabilmente analoga.

Tipo 136.1

Ampurias (Almagro 1955: 58 n. 8), a v. nera, alt. $15,5, \mathrm{n}$. dat.

\section{Tipo 136.2}

Ampurias (Almagro 1955: 204 n. 4), n. des., alt. 13,5 , età tiberiana;

Novaesum (Filtzinger 1972: tav. 46 n. 10), p. marrone, v. bruno rossiccia sull'intera superficie, alt. 16,8, 25-50 d.C.

\section{SERIE 137}

Corpo piriforme, collo allungato cilindrico leggermente svasato, orlo a tesa arrotondato, ampio fondo ben distinto.

\section{Tipo 137.1}

Ampurias (Almagro 1955: 59 n. 3), collo a v. nera, alt. 12,4, età augusteo-tiberiana.

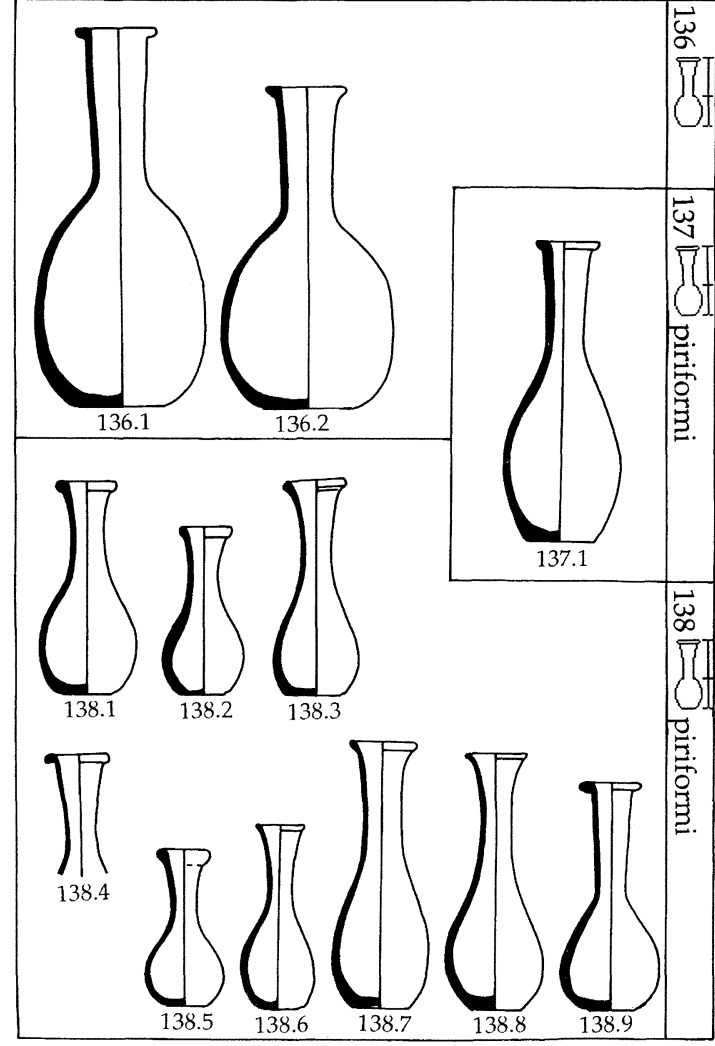

Fig. 10

\section{SERIE 138}

Corpo piriforme, collo allungato e strombato non ben distinto dal corpo, orlo estroflesso, arrotondato, talvolta ripiegato o tendente a fascetta sagomata, fondo piccolo più o meno distinto.

Serie dallo spettro cronologico piuttosto ampio; l'ampia diffusione del tipo e delle sue varianti comparata con le poco significative variazioni morfologiche porterebbe ad ipotizzare una destinazione rivolta verso contenuti piuttosto densi. L'evoluzione cronologica è ben evidente, dal prototipo 138.1 agli sviluppi successivi; il tipo 138.6 ha rapporti con il 132.6 e il 132.9.

\section{Tipo 138.1}

Castel d'Asso (Colonna 1970, tav. CCCCXIV, t. 36 , sottofacciata n. 2, 218), pasta giallo-rosata, collo a v. bruna, alt. 9,1, non stratificato;

Corinto (Blegen - Palmer - Young 1964: 167, 309 , pl. 99, 40a), ma con collo leggermente più allungato, p. arancio, collo sovradipinto, group i (tra il 44 a.C. e Augusto). 
Ostia (Zevi-Pohl 1970: 43, fig. 54), p. ocra, v. arancio-bruna su orlo e collo, alt. 7,7, fuori contesto.

\section{Tipo 138.2}

Veio (Torelli - Pohl 1973: 110, fig. 45 n. 230), p. nocciola chiaro, collo a v. nera, alt. $7,2, \mathrm{n}$. dat.;

Valentano (inedito), presso collezione privata notificata (informazione G. Gazzetti);

Torcello (Favaretto 1982: 177), p. rosa, tracce di v. rossa opaca, alt. 7, fuori contesto;

Pollentia (Arribas 1983: 321, 342, n. 40), p. grigia con anima arancio chiaro e zone fiammate, incompleto, fuori contesto;

Novaesum (Filtzinger 1972: tav. 46, n. 12), p. marrone, tracce di v. rosso-bruna sul corpo, 25-50 d.C.

\section{Tipo 138.3}

Ampurias (Almagro 1955: 49 n. 6), collo a v. nera, alt. 9, ultimi decenni del I secolo a.C.;

Sub Ascia (Passi Pitcher 1987: 44; 179,3), p. rossastra, acromo, alt. 9,2, contesto di età augustea.

\section{Tipo 138.4}

Padova (Zampieri 1991: 173; 191 n. 202), p. beige-rosato, fuori contesto.

\section{Tipo 138.5}

Ampurias (Almagro 1955: 59 n. 4), p. chiara, collo a v. nera, alt. 6,8 , in contesto augusteo-tiberiano.

\section{Tipo 138.6}

Ampurias (Almagro 1955: 188 n. 23), p. scura, alt. 7,9 , età tiberiana.

\section{Tipo 138.7}

Ampurias (Almagro 1955: 188 n. 15: 188 n. 17), in due esemplari; p. chiara, collo a v. nera, alt. 11 , età tiberiana; p. chiara, alt. 10, età tiberiana.

\section{Tipo 138.8}

Ampurias (Almagro 1955: 188 n. 14), p. chiara, collo a v. nera, alt. 10,6, età tiberiana.

\section{Tipo 138.9}

Ampurias (Almagro 1955: 68 n. 3), p. rossastra, collo a v. nera, alt. 8,5 , età claudio-neroniana.

\section{SERIE 139}

Corpo piriforme, collo allungato cilindrico, orlo a fascetta arrotondata più o meno sagomata, fondo piatto, ampio, più o meno distinto.

La serie deriva, o ha origine comune, con la 112.

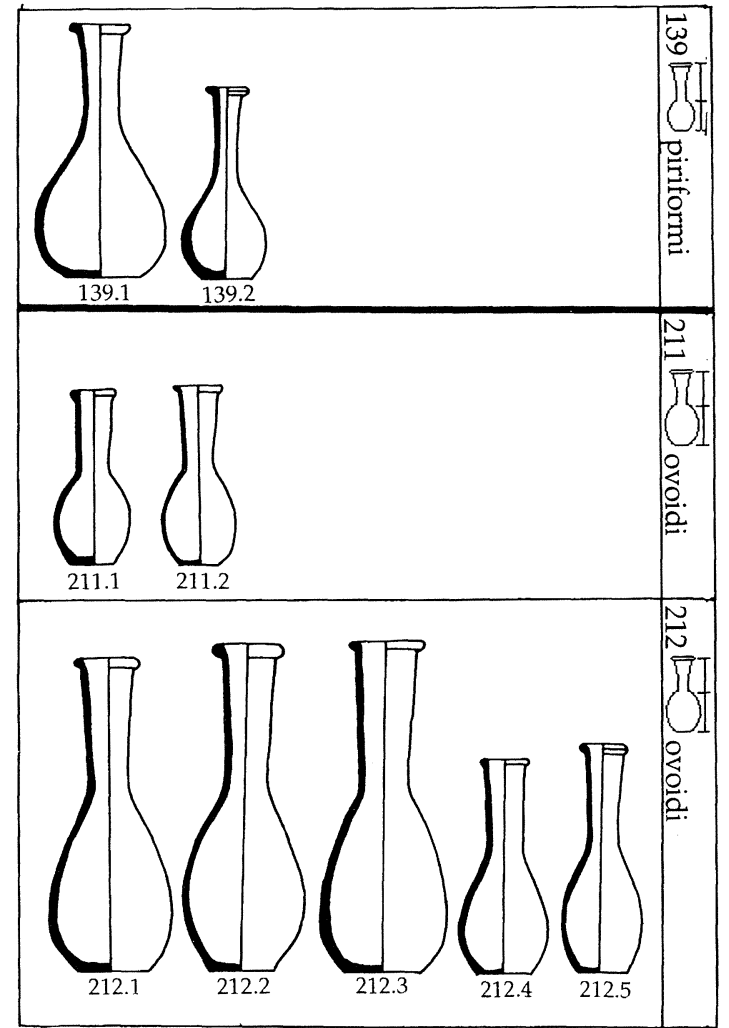

Fig. 11

Tipo 139.1

Ampurias (Almagro 1955: 46, nn. 4-9), in 6 esemplari; acromi, alt. 8,8, tra lo 0 e Tito.

Tipo 139.2

Ampurias (Almagro 1955: 49 n. 5), p. chiara, alt. 10, ultimi decenni del I secolo a.C.

\section{SERIE 211}

Di piccole dimensioni, collo cilindrico, orlo estroflesso più o meno ingrossato, piccolo fondo piatto più $o$ meno distinto.

\section{Tipo 211.1}

Ampurias (Almagro 1955: 285 n. 14), p. grigia, alt. 7,5, n. dat.

\section{Tipo 211.2}

Ampurias (Almagro 1955: 71 n. 7), p. rossastra, collo a v. nera, alt. $7,7, \mathrm{n}$. dat. 


\section{SERIE 212}

Corpo ovoide, collo cilindrico o leggermente troncoconico, orlo estroflesso e arrotondato, più o meno ingrossato, piccolo fondo piatto e ben distinto.

Serie piuttosto omogenea, che però consente scarsi appigli cronologici. I tipi dal 212.1 al 212.3 sono probabilmente antecedenti al 212.4 (tiberiano).

Tipo 212.1

Ampurias (Almagro 1955: 73 n. 3), p. rossastra alt. 13,3 , n. dat.

Tipo 212.2

Ampurias (Almagro 1955: 73 n. 2), p. rossastra, alt. 13,9 , n. dat.

Tipo 212.3

Ampurias (Almagro 1955: 73 n. 1), p. rossastra, alt. 14,2 , n. dat.

\section{Tipo 212.4}

Ampurias (Almagro 1955: 174 n. 4), p. chiara, tracce di v. sul collo, alt. 9,2, età tiberiana.

\section{Tipo 212.5}

Ampurias (Almagro 1955: 112 n. 4), resti di pittura, alt. 9,8, n. dat.

\section{SERIE 221}

Corpo globulare, collo cilindrico, piccolo orlo a tesa, fondo piatto più o meno distinto.

Serie di scarsa diffusione, da porre probabilmente nella prima metà del I secolo d.C.; è probabilmente dal 221.1 che deriva, tramite il 336.3 , la serie 336.

\section{Tipo 221.1}

Gabii (Vegas 1973: 154; 150 tipo 63,6), p. gialla, collo e interno a v. marrone scuro, prima metà del I secolo d.C.

\section{Tipo 221.2}

Ampurias (Almagro 1955: 84 n. 6), p. chiara, alt 8,4 , n. dat.

\section{SERIE 231}

Corpo piriforme, collo cilindrico strombato verso l'alto, orlo estroflesso più o meno ingrossato, talvolta ripiegato, fondo piatto ben distinto.

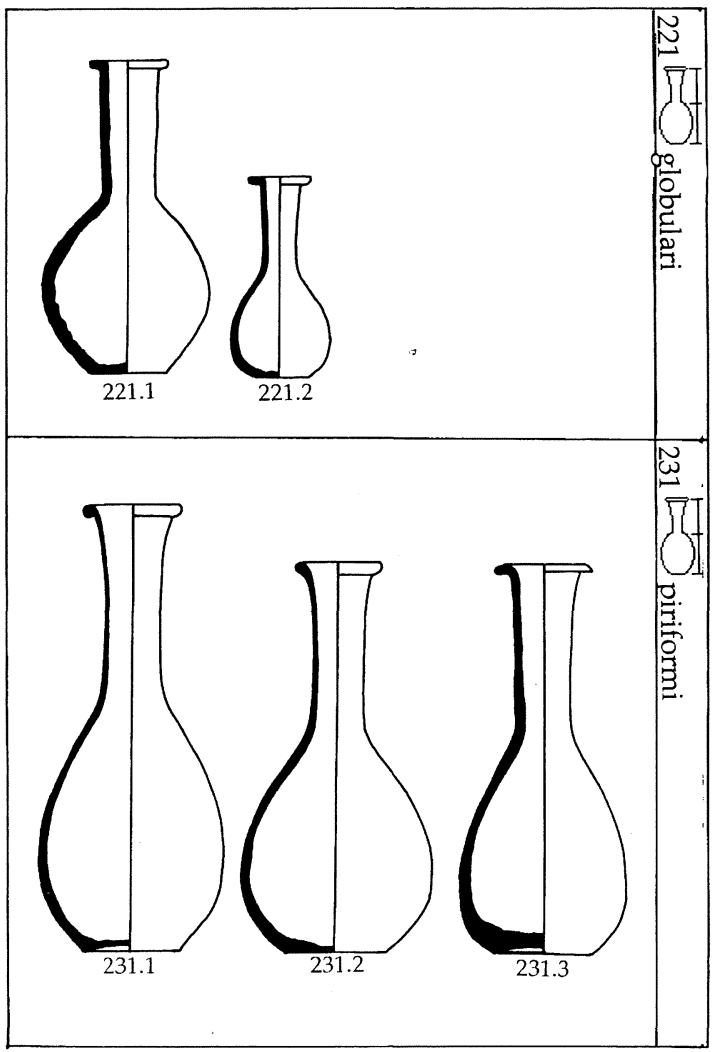

Fig. 12

Serie di ampia diffusione e di ampio spettro cronologico. I tipi 231.1-231.2-231.4 (e, probabilmente, il 231.3), sono i più antichi, compresi tra la tarda repubblica e la prima età augustea; le 231.5-7 sono le varianti successive.

\section{Tipo 231.1}

Stobi (Anderson 1977: 260, fig. 196 n. 758), v. bruno-rossiccia sul collo, senza datazione;

Taranto (E. Lippolis in De Juliis 1984: 513-514), p. arancio, v. nera opaca su orlo e collo, alt. 12,3, in contesto della seconda metà del I secolo a.C.;

Italia non meglio precisata (Hayes 1976: 33-4, 105 n. 157), p. marrone, ingubbiatura color seppia su collo e orlo, alt. 16, fuori contesto.

Tipo 231.2

Stobi (Anderson 1977: 260, fig. 196 n. 759), v. bruno-rossiccia sul collo, senza datazione;

Taranto (E. Lippolis in De Juliis 1984: 514), p. arancio, acromo, alt. 12, in contesto della seconda metà del I secolo a.C.;

Corinto (Blegen - Palmer - Joung 1964: 167, 294, pl. 78, 503.2/4), in 3 esemplari; i primi due in p. arancio, altezze $10,1-10,2$; il terzo in p. chiara 


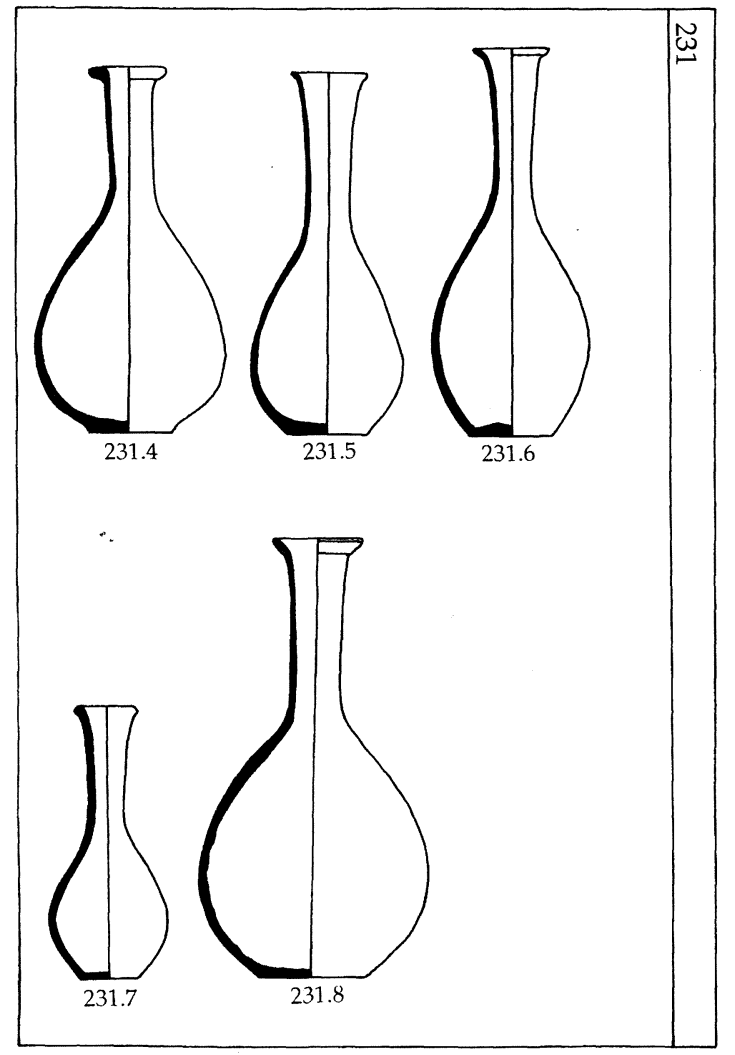

Fig. 13

molto porosa, alt. 10,3; «group i», tra il 44 a.C. e Augusto;

Ampurias (Almagro 1955: 204 n. 3), n. des., alt. 16,4 , età tiberiana.

\section{Tipo 231.3}

Stobi (Anderson 1977: 260, fig. 196 n. 760), v. bruno rossiccia sul collo, senza datazione.

Tipo 231.4

Ampurias (Almagro 1955: 49 n. 7), collo a v. nera, alt. 15 , prima età augustea.

\section{Tipo 231.5}

Ampurias (Almagro 1955: 166 n. 5), p. arancio, collo a v. scura, alt. $15, \mathrm{n}$. dat.

\section{Tipo 231.6}

Ampurias (Almagro 1955: $183 \mathrm{nn} .12-13$ : $194 \mathrm{n}$. 7), in tre esemplari; p. chiara, altezze 16-16,1, età tiberiano-claudia; con orlo indistinto, p. rossastra, alt. 16,3, età tiberiano-claudia.

\section{Tipo 231.7}

Ampurias (Almagro 1955: 255 n. 8), n. des., alt. 11,4 , età augusteo-tiberiana.

\section{Tipo 231.8}

Via Amerina (inedito), p. nocciola, v. rosso-bruna sul collo, alt. 18,2 , contesto augusteo.

\section{SERIE 232}

Di piccole dimensioni, corpo piriforme compresso, tendente alla carena e con cambi di curvatura piuttosto netti, collo troncoconico, orlo ingrossato più o meno distinto, a volte scanalato internamente, fondo piatto poco distinto.

Non antecedenti il primo secolo d.C., sono imitazioni di forme in vetro; per questa serie, come per altre successive, valgono le osservazioni alla serie 233.

\section{Tipo 232.1}

Stobi (Anderson 1977: 261, fig. 199 n. 773), v. bruno-rossiccia sul collo, $n$. dat.;

Gropello Cairoli (PV) (Fortunati Zuccala 1979: 50), p. arancio, alt. 5,5, associato con moneta di Tiberio del 22 d.C.

\section{Tipo 232.2}

Stobi (Anderson 1977: 261, fig. 199 n. 774), v. bruno-rossiccia sul collo, $\mathrm{n}$. dat.

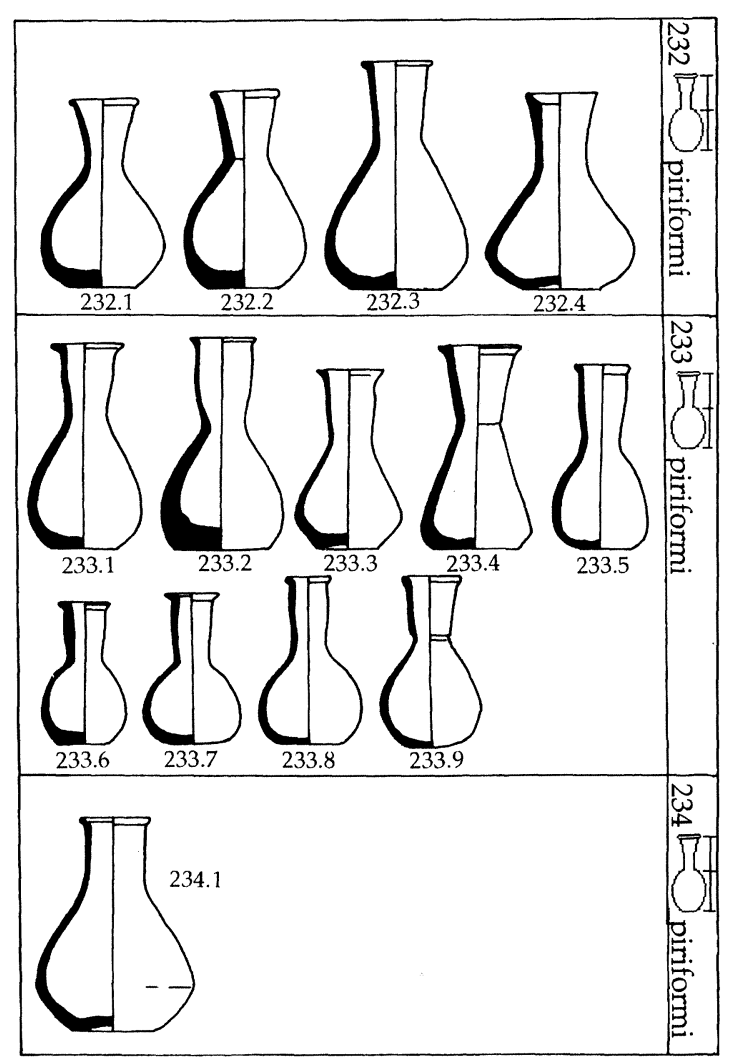

Fig. 14 
Tipo 232.3

Stobi (Anderson 1977: 261 fig. 202 n. 779), v. bruno-rossiccia sul collo, n. dat.;

Ampurias (Almagro 1955: $197 \mathrm{nn} .11-14-16-17$ ), in quattro esemplari; n. des., alt. 8,4; n. des., alt. 8,5 ; n. des., alt. 7,7; n. des., alt. 7,6; n. dat.

Tipo 232.4

Stobi (Anderson 1977: 261, fig. 202 n. 780), v. bruno-rossiccia sul collo, n. dat.;

Corinto (Blegen - Palmer - Young 1964: 167, 295, pl. 76, 506-5), p. arancio, collo dipinto, alt. 9,9, «group ii», augusteo.

\section{SERIE 233}

Di piccole dimensioni, corpo piriforme compresso, con carena più o meno evidente, con cambi di curvatura generalmente netti, collo troncoconico distinto dal corpo da una strozzatura più o meno accentuata, orlo ingrossato a sezione triangolare, ampio fondo piatto più o meno distinto.

Serie di tipi che tendono ad imitare i balsamari vitrei. La strozzatura della spalla e la tendenza al collo svasato, oltre che i contesti di rinvenimento, databili però solo per i tipi 233.5 e 233.6, fanno propendere per una datazione al I secolo d.C., in un periodo in cui il vasellame in vetro comincia ad imporsi prepotentemente sul mercato; ne deriva la nascita di forme ad imitazione del vetro: $233.6 \mathrm{e}$ 233.7 sono evidentemente derivati dalla forma palestinese 237.1, ma sono di produzione locale (ispanica, in questo caso); hanno comunque stretti rapporti con i 233.7 e 237.2 .

Tipo 233.1

Stobi (Anderson 1977: 261, fig. 202 n. 777), v. bruno-rossiccia su collo, n. dat.

\section{Tipo 233.2}

Stobi (Anderson 1977: 261, fig. 202 n. 776), v. bruno-rossiccia sul collo, n. dat.

Tipo 233.3

Stobi (Anderson 1977: 261, fig. 199 n. 775), v. bruno-rossiccia sul collo, n. dat.;

Ostia (Zevi - Pohl 1970: 165, fig. 76, 118), mancante del bordo, p. nocciola-arancio, v. rosa-violacea sul collo, n. dat.

Tipo 233.4

Stobi (Anderson 1977: 261, fig. 199 n. 772), v. rosso-bruna sul collo, n. dat.
Tipo 233.5

Ampurias (Almagro 1955: 182 n. 5; 204 n. 7; 246 n. 3; 246 n. 4; 275 n. 68), in cinque esemplari; p. scura, alt. 7,6, dallo 0 all' età tiberiana; n. des., alt. 7,8, età tiberiana; n. des., alt. 8,2, n. dat.; con orlo sagomato, n. des., alt. 7,9, n. dat.; con orlo appiattito superiormente, v. nera sul collo, alt. 7,8, n. dat.

\section{Tipo 233.6}

Ampurias (Almagro 1955: 111 n. 1; 275 n. 70; 109 n. 13; 282 n. 3; 272 n. 5), collo a v. nera, alt. 5,8 , dallo 0 all'età tiberiana; di resa più rozza, con pareti più spesse, a v. nera sul collo, alt. 7,4, n. dat.; analogo al precedente, tracce di v. rossa sul corpo, alt. 6,4, n. dat.; analogo ai precedenti, n. des., alt. 6, n. dat.; analogo ai precedenti e deformato, n. des., alt. $6,2, n$. dat.

\section{Tipo 233.7}

Ampurias (Almagro 1955: 68 n. 11), p. grigia, alt. $6,3, \mathrm{n}$. dat.

\section{Tipo 233.8}

Ampurias (Almagro 1955: 197 n. 8), n. des., alt. 7, n. dat.

Tipo 233.9

Ampurias (Almagro 1955: 79 n. 4), p. rossastra, alt. 7,1, n. dat.

\section{SERIE 234}

Di piccole dimensioni, corpo piriforme compresso e carenato, collo cilindrico, orlo ingrossato leggermente distinto, con scanalatura interna, ampio fondo piatto distinto.

Tipo 234.1

Stobi (Anderson 1977: 261, fig. 202 n. 782), v. rosso-bruna sul collo, n. dat.

\section{SERIE 235}

Di piccole dimensioni, corpo piriforme compresso, collo cilindrico più o meno strombato verso l'alto, orlo estroflesso arrotondato, talvolta leggermente ingrossato o appiattito superiormente, ampio fondo piatto più o meno distinto.

Altra serie nata dall'imitazione delle forme in vetro. 


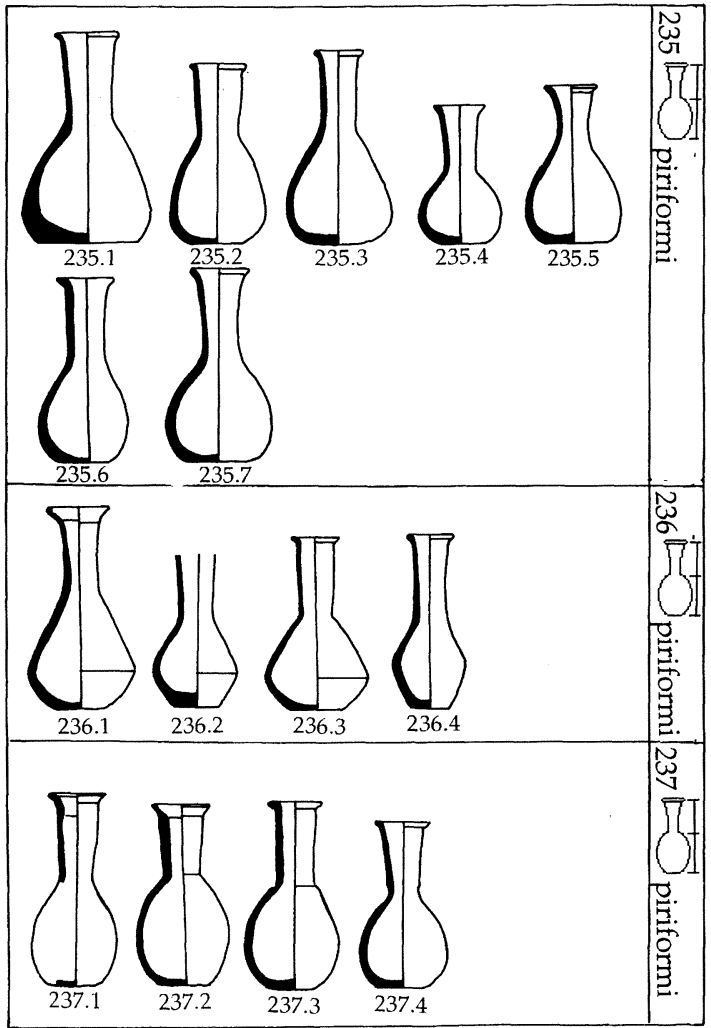

Fig. 15

Tipo 235.1

Stobi (Anderson 1977: 261, fig. 202, n. 772), v. rosso-bruna sul collo, $\mathrm{n}$. dat.

Tipo 235.2

Ampurias (Almagro 1955: 182 n. 2; 182 n. 3), in due esemplari; p. scura, alt. 7,6, dallo 0 all'età tiberiana; con corpo più rigonfio, $\mathrm{n}$. des., alt. $8, \mathrm{n}$. dat.

Tipo 235.3

Ampurias (Almagro 1955: 197 n. 10; 185 n. 12), in due esemplari; n. des., alt. 8,1, n. dat.; p. chiara, alt. 9, età tiberiano-claudia.

Tipo 235.4

Ampurias (Almagro 1955: 228 n. 1; 185 n. 13), in due esemplari; n. des., alt. 5,8, n. dat.; p. chiara, alt. 6 , età tiberiano-claudia.

\section{Tipo 235.5}

Ampurias (Almagro 1955: 103 n. 9; 93 n. 3), in due esemplari; n. des., alt. 6,7, dallo 0 all'età tiberiana; con orlo più ingrossato internamente, collo con tracce di v., dallo 0 all'età tiberiana.
Tipo 235.6

Ampurias (Almagro 1955: 268 n. 12), p. grigia, alt. 7,7 , n. dat.

Tipo 235.7

Ampurias (Almagro 1955: 197 n. 13), n. des., alt. 8,2 , n. dat.

\section{SERIE 236}

Corpo piriforme carenato, collo cilindrico, orlo estroflesso troncoconico più o meno distinto, talvolta semplicemente arrotondato, fondo piatto.

Serie di imitazione di forme di importazione orientale; vedi il commento alla serie 237 .

Tipo 236.1

Stobi (Anderson 1977: 260, fig. 199 n. 769), v. rosso-bruna sul collo, n. dat.

Tipo 236.2

Padova (Zampieri 1991: 173: 191 n. 203), incompleto, p. gialla-rosata, fuori contesto.

Tipo 236.3

Ampurias (Almagro 1955: 181 n. 10), p. rossiccia, collo a v. arancio scura, alt. 7,3, età tiberianoclaudia.

Tipo 236.4

Ampurias (Almagro 1955: 188 nera 16), p. scura, alt. 7,4, etá tiberiana.

\section{SERIE 237}

Corpo piriforme piuttosto rigonfio, collo cilindrico o tronco-conico generalmente distinto dal corpo, orlo estroflesso, inclinato, talvolta ingrossato, fondo piatto poco distinto.

Serie di produzione orientale (la 237.1, forse la 237.2) o di imitazione locale (337.3); di derivazione molto più tarda (età claudio-neroniana) la 237.4.

\section{Tipo 237.1}

Palestina non meglio precisata (Hayes 1976: 62, 84 n. 338), alt. 8,3, p. arancio-rosata, secondo l'Editore della prima metà del I secolo d.C.;

Emona (Plesnicar - Gec 1972: t. 291), con orlo simile a quello della serie 233 , p. rosso grigiastra, alt. 15, contesto di I sec. a.C.

\section{Tipo 237.2}

Ampurias (Almagro 1955: 160 n. 23), v. nera su tutta superficie, alt. 7,7, età tiberiano-claudia. 
Tipo 237.3

Ampurias (Almagro 1955: 185 n. 8), p. chiara, alt. 7,9, età tiberiano-claudia.

Tipo 237.4

Ampurias (Almagro 1955: 165 n. 1), a v. nera sul'intera superficie, alt. $5,1, \mathrm{n}$. dat.

\section{SERIE 238}

Corpo piriforme, collo strombato distinto nettamente dal corpo, orlo indistinto arrotondato, ampio fondo piatto ben distinto.

Altro balsamario di produzione orientale (palestinese), come il 237.1.

\section{Tipo 238.1}

Palestina non meglio precisata (Hayes 1976: 62, n. 342 ), alt. 11,2 , p. arancio-marrone, tracce di v. rosso-bruna sul collo, secondo l'Editore della prima metà del I secolo d.C.

\section{SERIE 239}

Corpo piriforme, collo troncoconico strombato verso l'alto, poco distinto dal corpo, orlo estroflesso, più o meno ingrossato e arrotondato, talvolta sagomato $\mathrm{o}$ a fascetta arrotondata, fondo più o meno distinto.

Serie piuttosto ben definita morfologicamente, ma che consente scarsi appigli cronologici. Si tratta comunque probabilmente di produzioni del I secolo d.C.

\section{Tipo 239.1}

Ampurias (Almagro 1955: 88 n. 1), p. rossastra, alt. $12,1, \mathrm{n}$. dat.

\section{Tipo 239.2}

Ampurias (Almagro 1955: 70 n. 8), p. rossastra, alt. 9,5 , n. dat.

Tipo 239.3

Ampurias (Almagro 1955: 188 n. 11), p. chiara, alt. 15,6 , età tiberiana.

\section{Tipo 239.4}

Ampurias (Almagro 1955: 188 n. 18; 197 n. 9; 74 n. 2), in tre esemplari; p. chiara, alt. 9,9, età tiberiana; n. des., alt. 8,7, n. dat.; con orlo a fascetta, p. scura, collo con tracce di v., alt. 8,5, n. dat.

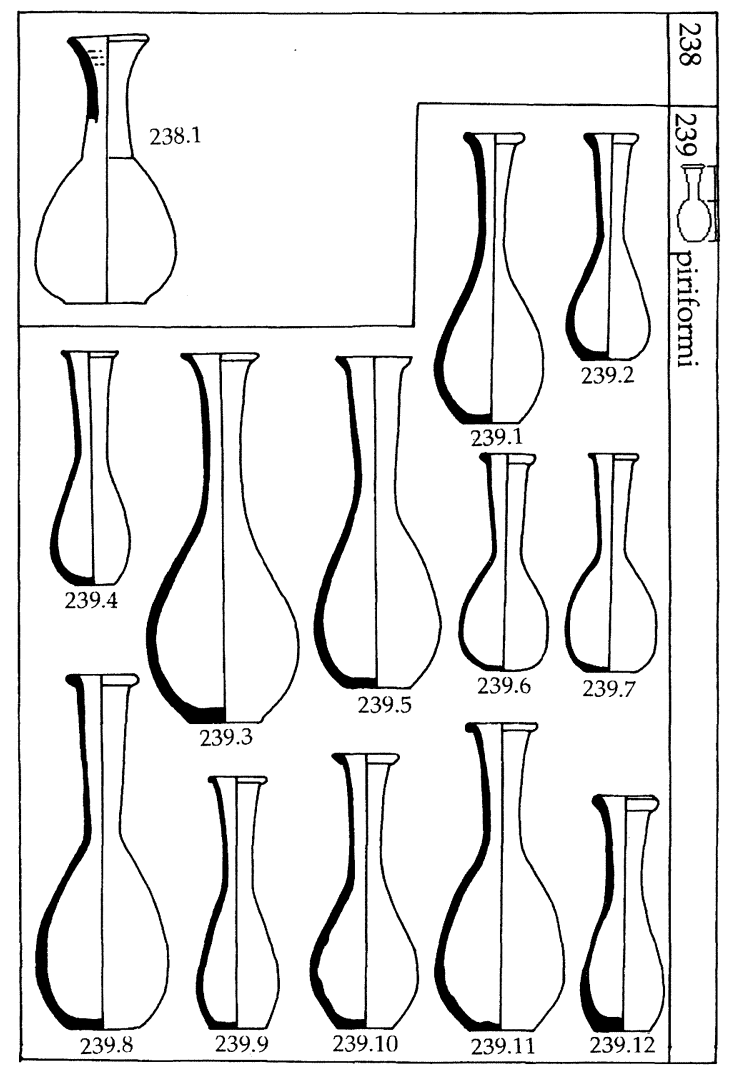

Fig. 16

Tipo 239.5

Ampurias (Almagro 1955: 166 n. 6), p. arancio, collo a v. scura, alt. 14,1, n. dat.

Tipo 239.6

Ampurias (Almagro 1955: 71 n. 5; 229 n. 7), in due esemplari; p. rossastra, collo a v. nera, alt. 9,2, $\mathrm{n}$. dat.; con orlo leggermente ripiegato, $\mathrm{n}$. des., alt. 10 , n. dat.

Tipo 239.7

Ampurias (Almagro 1955: 71 n. 4), p. rossastra, collo a v. nera, alt. 9,3, n. dat.

Tipo 239.8

Ampurias (Almagro 1955: 84 n. 2), p. chiara, alt. 15 , n. dat.;

Via Amerina (inedito), p. nocciola, v. rosso-bruna sul collo, alt. 11,5, contesto augusteo.

\section{Tipo 239.9}

Ampurias (Almagro 1955: 174 n. 2), p. rossiccia, collo con tracce di v. , alt. 10,7, età tiberiana. 


\section{Tipo 239.10}

Via Amerina (inedito), p. nocciola, v. rosso-bruna sul collo, alt. 11,5, contesto augusteo.

Tipo 239.11

Via Amerina (inedito), p. nocciola, v. rosso-bruna sul collo, alt. 12,8, contesto augusteo.

Tipo 239.12

Ampurias (Almagro 1955: 105 n. 8), con resti di v. sulla superficie, alt. 10, augusteo.

\section{SERIE 311}

Balsamario (?) di grandi dimensioni, corpo ovoide, ampio collo cilindrico, orlo a sezione triangolare, arrotondato esternamente e appiattito superiormente.

Posto come balsamario dalla Anderson (1977: 261) per il contesto di rinvenimento, è forse un recipiente da trasporto per essenze.

Tipo 311.1

Stobi (Anderson 1977: 261, fig. 202 n. 784), v. rossa sull'intera superficie esterna, incompleto, $n$. dat.

\section{SERIE 312}

Corpo ovoide allungato, corto collo leggermente troncoconico poco distinto dal corpo, orlo estroflesso arrotondato, piccolo fondo piatto poco distinto.

Tipo 312.1

Ampurias (Almagro 1955: 229 n. 3), non descitto, alt. 11, n. dat.;

Emona (Plesnicar - Gec 1972: t. 191), con il corpo più cilindrico e l'orlo leggermente strombato, $p$. rossa, alt. 17,2, contesto di I sec. d.C.

\section{SERIE 321}

Corpo globulare compresso, corto e ampio collo cilindrico, orlo leggermente estroflesso, inclinato internamente, fondo concavo ben distinto.

Unica attestazione di questa forma a collo largo, che però ha evidenti rapporti con le serie 232 e 233, e soprattutto con il tipo 234.1. La datazione proposta, per i medesimi motivi precedentemente esposti, non può essere più alta dell'età tiberiana.

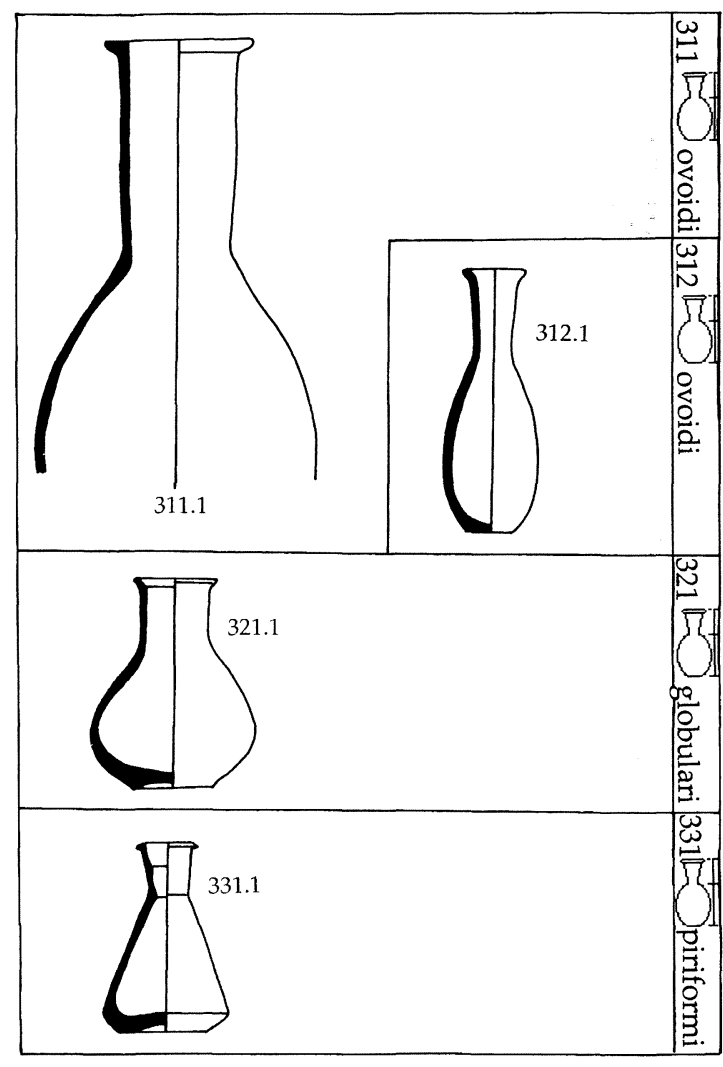

Fig. 17

Tipo 321.1

Stobi (Anderson 1977: 261, fig. 202 n. 780), v. rosso-bruna sul collo, n. dat.

\section{SERIE 331}

Corpo «piriforme» quasi troncoconico con bassa carena ben marcata, corto collo troncoconico con orlo ripiegato a sezione triangolare, ampio fondo piatto ben distinto.

\section{Tipo 331.1}

Stobi (Anderson 1977: 261, fig. 199 n. 771), p. rosso-bruna, v. bruna su collo, n. dat.

\section{SERIE 332}

Corpo piriforme allungato, corto collo poco distinto, strozzato e strombato verso l'alto, orlo poco distinto, più o meno ingrossato e arrotondato, piccolo fondo poco distinto. 


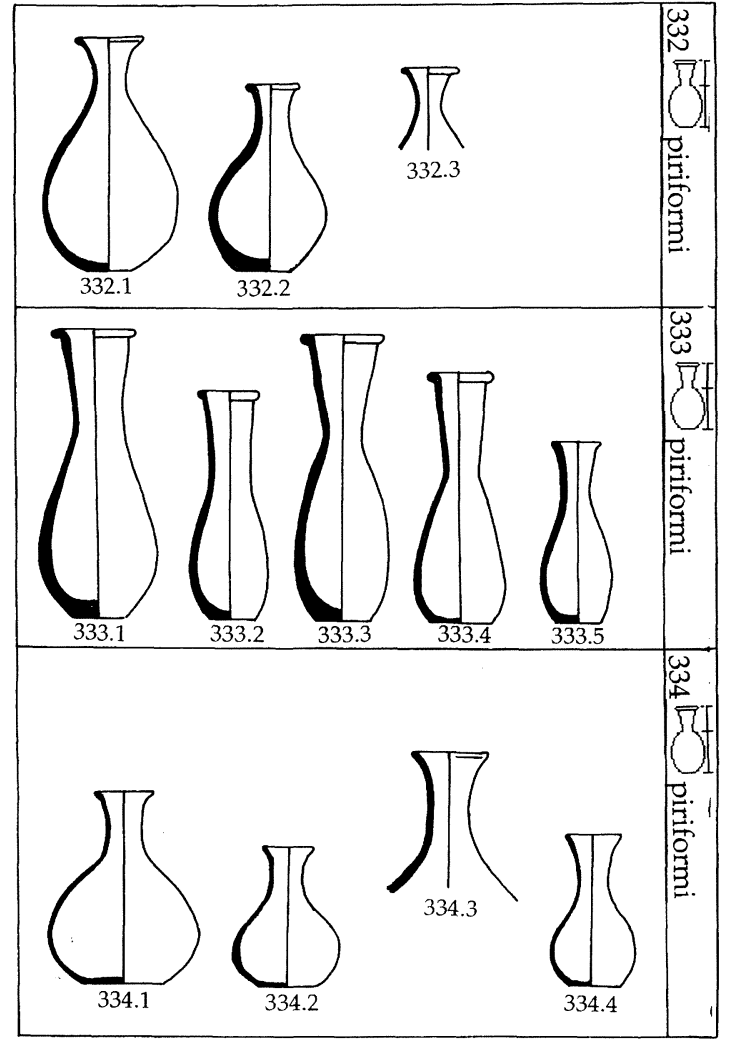

Fig. 18

Serie con una cronologia compresa tra l'età augustea e tutta la prima metà del I secolo d.C.; la serie ha stretti raporți con la 334, particolarmente con il tipo 334.4; la tendenza alla atrofizzazione dell'orlo è caratteristica comune di molte di queste serie; nella maggior parte dei casi si tende ad un orlo indistinto su collo a tromba come nel caso del tipo 332.1; fortemente improbabile la datazione proposta dalla Schojer (cfr. infra) per questo tipo.

\section{Tipo 332.1}

Stobi (Anderson 1977: 260 fig. 199 n. 770), p. rossa, v. rossa sull'intera superficie, $n$. dat.;

Taranto (T. Schojer in De Juliis 1984: 511), in due esemplari; con corpo leggermente più convesso, p. arancio, ingubbiatura arancio, v. nera su orlo e collo con colature, alt. 11,2; p. rosa arancio, ingubbiatura arancio, v. rossa opaca su orlo e collo, alt. 10,1 , contesto della prima metà del I secolo d.C.

Tipo 332.2

Pollentia (Vegas 1973: 154; 150 tipo 63,8), p. giallo scura, v. bruno-rossiccia su collo e internamente, prima metà del I secolo d.C.

\section{Tipo 332.3}

Lucus Feroniae (inedito), p. grigia, v. nera opaca su collo e internamente, contesto presumibilmente augusteo.

\section{SERIE 333}

Corpo piriforme allungato, corto collo troncoconico piuttosto ampio, generalmente poco distinto dal corpo, orlo estroflesso, più o meno distinto, tendente alla fascetta arrotondata, piccolo fondo ben distinto.

Il prototipo, anche se n. dat., è probabilmente il 333.1 , forse da porre, come il 333.2, nella prima età augustea. Nella 333.5 si osserva la caratteristica atrofizzazione dell'orlo tipica delle ultime produzioni.

\section{Tipo 333.1}

Stobi (Anderson 1977: 260, fig. 199 n. 767), v. rosso-bruna sul collo, n. dat.

\section{Tipo 333.2}

Ampurias (Almagro 1955: 58 n. 5), v. rosso-nerastra su collo, alt. 9,8, associato con moneta del 42 a.C.

\section{Tipo 333.3}

Ampurias (Almagro 1955: 65, n. 1), collo a v. nera, alt. 12, dallo 0 a Tiberio;

Vindonissa (Ettlinger - Simonett 1952, t. 11), acromo, alt. 15,6, I sec. d.C.

\section{Tipo 333.4}

Ampurias (Almagro 1955: 73 n. 4), p. rossastra, collo a v. rossa, alt. 10,5 , n. dat.

\section{Tipo 333.5}

Ampurias (Almagro 1955: 196 n. 22), n. des., alt. 7,6 , età tiberiana.

\section{SERIE 334}

Corpo piriforme compresso, corto collo strombato, orlo tendenzialmente indistinto, più $o$ meno estroflesso, fondo piatto generalmente ampio e poco distinto.

Serie con caratteristiche analoghe a quelle già commentate per la 332; qui, nello stesso contesto di Portorecanati, assistiamo alle fasi finali della evoluzione, che passa per i tipi 335.1-2. Il tipo 334.5 ha stretti rapporti con il 335.4 ; il 334.7 con il 113.5 . Il 


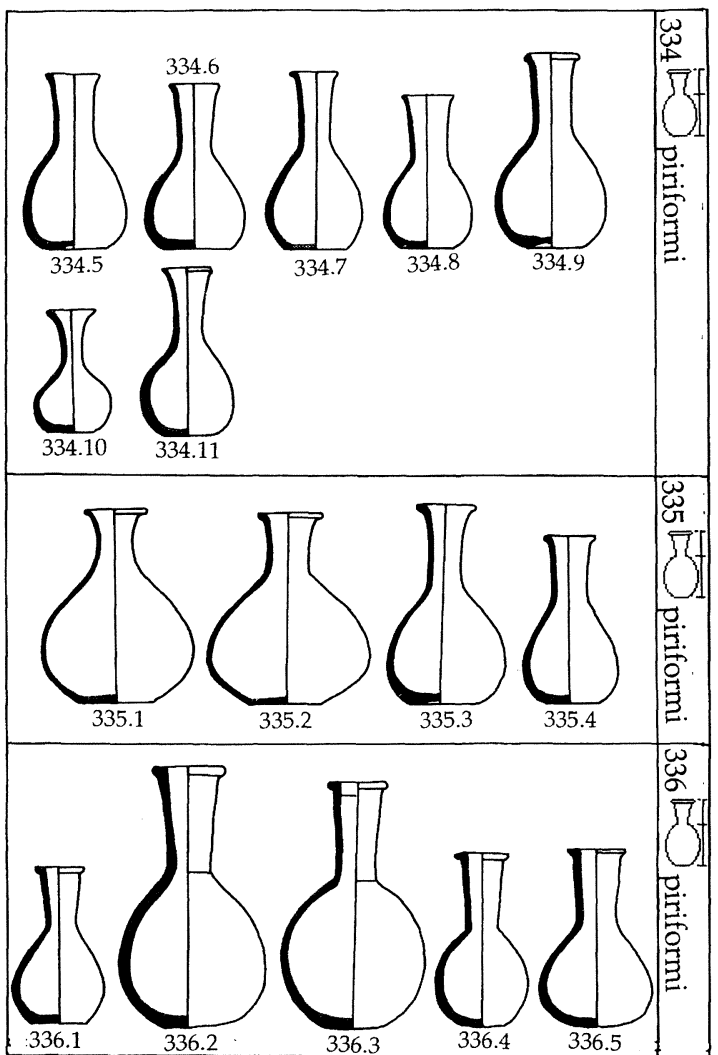

Fig. 19

334.10 è l'estremizzazione del 334.4. Il 334.11 ha rapporti con i tipi 121.1.e 113.4 .

\section{Tipo 334.1}

Portorecanati (Mercando 1974: 404, fig. 328 h), p. ocra, v. marrone chiaro sul collo, alt. 8,4 , dalla fine del I secolo a.C. alla prima metà del I secolo d.C.

Tipo 334.2

Portorecanati (Mercando 1974: 404, fig. 328a), p. ocra, v. bruna su collo, p. $6 \mathrm{~cm}$, dalla fine del I secolo a.C. alla prima metà del I secolo d.C.

\section{Tipo 334.3}

Portorecanati (Mercando 1974: 404, fig. 328e), p. ocra, v. marrone diluita, incompleto, dalla fine del I secolo a.C. alla prima metà del I secolo d.C.

\section{Tipo 334.4}

Castel D'Asso (Colonna 1970: tav. CCCCXIV, t. 36 sottofacciata n. 3: 218), pasta giallo-rosata, acromo, alt. 6,6 , non stratificato;
Ampurias (Almagro 1955: 194 n. 9; 196 n. 24), in due esemplari; p. chiara, alt. 6,4, età tiberianoclaudia; n. des., alt. 7,7, età tiberiana.

\section{Tipo 334.5}

Ampurias (Almagro 1955: 196 n. 20; 196 n. 18; 196 n. 13), in tre esemplari; n. des., alt. 7,2, età tiberiana; n. des., alt. 7, età tiberiana; con collo leggermente più allungato, alt. 7,4, età tiberiana.

\section{Tipo 334.6}

Ampurias (Almagro 1955: 196 n. 14; 196 n. 23), in due esemplari; n. des., alt. 7 , età tiberiana; $n$. des., alt. 7,6, età tiberiana.

\section{Tipo 334.7}

Ampurias (Almagro 1955: 195 n. 4; 196 n. 19; 92 n. 7; 88 n. 2), in quattro esemplari; n. des., alt. 7,5 , n. dat.; n. des., alt. 6,1 , età tiberiana; con orlo appiattito superiormente, tracce di v. sul collo, alt. 9,3 , età tiberiano-claudia; analogo al precedente, $\mathrm{p}$. grigia, n. dat., alt. 9,6.

\section{Tipo 334.8}

Ampurias (Almagro 1955: 195 n. 3; 240 n. 3), in due esemplari; n. des., n. dat., alt. 6,2; con parete del collo più spessa in sezione, alt. 3,6, età augusteo-tiberiana.

\section{Tipo 334.9}

Ampurias (Almagro 1955: 188 n. 24; 185 n. 8), p. scura, alt. 8,2 , età tiberiana; con orlo leggermente più arrotondato, p. chiara, età tiberiano-claudia.

Tipo 334.10

Ampurias (Almagro 1955: 188 n. 12), p. rossastra, collo a v. giallastra, alt. 5,1, età tiberiana.

Tipo 334.11

Ampurias (Almagro 1955: 188 n. 13), p. scura, collo a v. giallastra, età tiberiana.

\section{SERIE 335}

Corpo piriforme compresso, corto collo strombato, orlo estroflesso leggermente ingrossato e arrotondato, fondo piatto generalmente poco distinto.

Vedi commento della serie 334 .

\section{Tipo 335.1}

Portorecanati (Mercando 1974b: 404, fig. 328d), p. ocra, tracce di v. bruno-chiara sul collo, alt. 8,4 , dalla fine del I secolo a.C. alla prima metà del I secolo d.C. 


\section{Tipo 335.2}

Portorecanati (Mercando 1974b: 403, fig. 328f), p. fine, color ocra, tracce di v. bruna su orlo, alt. 8,4 , dalla fine del I secolo a.C. alla prima metà del I secolo d.C.

\section{Tipo 335.3}

Vico Equense (Bonghi Jovino 1982: 78, tav. $117,5)$, p. camoscio-grigiastra, v. nera opaca su bocchello, alt. 8,8 , fuori contesto.

\section{Tipo 335.4}

Ampurias (Almagro 1955: $197 \mathrm{nn} .12-15)$, in due esemplari; non descritti, altezze $8,2-8,4$, n. dat..

\section{SERIE 336}

Corpo piriforme molto espanso, tendente al globulare, corto collo troncoconico, orlo estroflesso tendenzialmente appiattito superiormente e arrotondato all'esterno, fondo piatto per lo più piccolo, più o meno distinto.

Serie piuttosto omogenea cronologicamente. Forse più antico (prima età augustea?) il 236.3.

\section{Tipo 336.1}

Pollentia (Arribas 1983: 326, 345, n. 70), p. bruno-rossiccia, fine e ben decantata, alt. 6,7 , contesto tiberiano-flavio;

Padova (Zampieri 1991: 173; 191 n. 199), mancante di orlo, p. arancio-rosata, fuori contesto;

Italia non meglio precisata (Hayes 1976: 34; 104 n. 158), alt. 4,8, p. marrone, v. rosso-bruna, fuori contesto;

Ampurias (Almagro 1955: 93 n. 4; 92 n. 5), collo con tracce di pittura, alt. 7,4, n. dat.; con tracce di v. sul collo, alt. 7,8 , età tiberiano-claudia.

Tipo 336.2

Sassari (Maetzke 1964: 304, 307 n. 1), alt. 10,8 acromo, n. dat.

Tipo 336.3

Bolsena (Santrot et al. 1992: 45, fig. 3 n. 509), p. grigia, ingubbiato, alt. 16,1 .

\section{Tipo 336.4}

Ampurias (Almagro 1955: 275 n. 67; 108 n. 2; 185 n. 10; 285 n. 20; 69 n. 8), in cinque esemplari; v. nera sul collo, alt. 7,4, n. dat.; n. des., alt. 7,4, primo venticinquennio del I secolo d.C.; p. chiara, alt. 7,2, età tiberiano-claudia; p. grigia, n. dat., alt.
6,3; con strozzatura appena accennata all'attaccatura del collo, p. rossastra, collo a v. nera, 2 incisioni parallele alla base del collo, alt. $8, \mathrm{n}$. dat.

Tipo 336.5

Ampurias (Almagro 1955: 92 n. 6; 268 n. 10; 268 n. 13; 185 n. $11 ; 108$ n. 1), in cinque esemplari; tracce di v. sul collo, alt. 7,4, contesto età tiberianoclaudia; p. grigia, alt. 6,4, n. dat.; p. chiara, v. rossa su collo, alt. 7,8, n. dat.; con fondo con stacco molto più netto, p. chiara, alt. 7,4 , contesto di età tiberiano-claudia; n. des., alt. 7,8, primo venticinquennio del I secolo d.C.

\section{Tipo 336.6}

Ampurias (Almagro 1955: 79 n. 2; 71 n. 8), in due esemplari; non descritta, alt. 8,4, n. dat.; p. rossastra, collo a v. nera, alt. $5,1, \mathrm{n}$. dat.

\section{Tipo 336.7}

Ampurias (Almagro 1955: 103 n. 8; 103 n. 10; 103 n. 11; 97 n. 32), in quattro esemplari; n. des., alt. 7,4 , tra lo 0 e l'età tiberiana; n. des., alt. 5,5, tra lo 0 e l'età tiberiana; con collo leggermente più al-

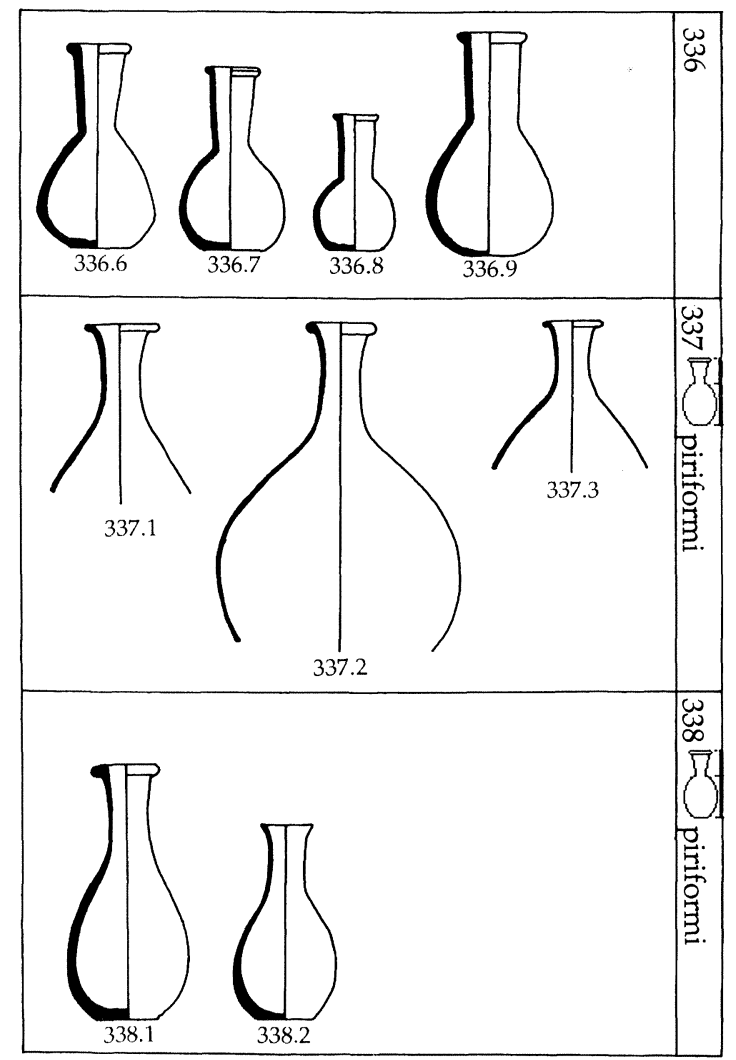

Fig. 20 
lungato, n. des., alt. 5,6, tra lo 0 e l'età tiberiana; con orlo arrotondato, n. des., alt. 7, n. dat.; n. des., alt. 8,6, ultimo venticinquennio del I secolo a.C.

\section{Tipo 336.8}

Ampurias (Almagro 1955: 186 n. 9), p. chiara, collo a v. rossa, $n$. dat.

Tipo 336.9

Ampurias (Almagro 1955: 81 n. 5; 79 n. 3), in due esemplari; p. scura, alt. 9,2, n. dat.; n. des., alt. 5,6, n. dat.

\section{SERIE 337}

Corpo piriforme molto espanso, corto collo strombato, più o meno strozzato nella parte mediana e poco distinto dal corpo, orlo ingrossato e ripiegato, arrotondato.

Serie di scarsa diffusione (probabilmente di produzione dell'area tiberina) e di cronologia piuttosto ben definita all'età augustea. Ha comunque rapporti con la serie 332 .

Tipo 337.1

Lucus Feroniae (inedito), p. arancio, v. rossobruna su collo e internamente, contesto presumibilmente augusteo.

Tipo 337.2

Lucus Feroniae (inedito), p. rosa-grigiastra, v. bruno-nerastra evanida su collo e internamente, contesto presumibilmente augusteo;

Lilibeo (Bisi 1970: 529, fig. 18b), con collo leggermente più corto e sempre senza fondo, dimensioni analoghe, p. ocra, v. nera opaca iridescente internamente, lisciato a stecca esternamente, $v$. anche su orlo e collo, in contesto di I secolo a.C. non meglio definibile.

\section{Tipo 337.3}

Lucus Feroniae (inedito), p. arancio, v. rossabruna su collo e internamente, contesto presumibilmente augusteo.

\section{SERIE 338}

Corpo piriforme, corto collo cilindrico poco distinto dal corpo, orlo ripiegato, ingrossato e arrotondato o indistinto e strombato, fondo piatto ben marcato.
Tipo 338.1

Ampurias (Almagro 1955: 57 n. 12), p. grigia, collo a v. nera, ultimi decenni del I secolo a.C.

Tipo 338.2

Ampurias (Almagro 1955: 235 n. 7; 177 n. 12), in due esemplari; n. des., alt. 8 , n. dat.; n. des., incompleto, n. dat.

\section{BIBLIOGRAFIA}

Albrecht, C., 1938: Das Römerlager in Oberaden, Dortmund.

Almagro, M., 1955: Las Necrópolis de Ampurias, vol. II, Barcelona.

Anderson, V. R., 1977: Pottery of the late Hellenistic and early Roman periods at Stobi, Tesi di Laurea, University Microfilm International.

Anderson Stojanovich, V. R., 1987: The chronology and function of ceramic unguentaria, American Journal of Archaeology, 91.1, 105-122.

Arribas, A., 1983: 3. Pollentia, Estudio de los Materiales, 1. Sa Portella. Excavaciones 19571963, Palma de Mallorca.

Bisı, A. M., 1970: Lilibeo (Marsala). Scavi nella necropoli dei Cappuccini, Notizie Scavi, XXIV, 524-559.

Blegen, C. W. - Palmer, H. - Young, R. S., 1964: Corinth XIII, the north cemetery, Princeton, Amer. School of Class. Stud. at Athens.

Bonghi Jovino, M., 1982: La necropoli preromana di Vico Equense, Cava dei Tirreni.

Bonghi Jovino, M., 1986: Gli Etruschi di Tarquinia, Modena.

Brecciaroli Taborelli, L., 1990: Segusio: nuovi dati ed alcune ipotesi, Quaderni della Soprintendenza Archeologica del Piemonte, 9. 65-158.

Bruneau, P., 1970: Tombes d'Argos, Bulletin de Correspondence Hellénique, 69, 437-531.

Colonna, G., - Di Paolo Colonna, E., 1970: Le necropoli rupestri dell'Etruria Meridionale-Castel D'Asso, Roma.

Colonna, G., - Di Paolo Colonna, E., 1978: Le necropoli rupestri dell'Etruria Meridionale-Norchia, Roma.

De JuliIs, E. (a cura di), 1984: Gli Ori di Taranto in età ellenistica, Catalogo della Mostra (Milano, dicembre 1984 - marzo 1985), Milano.

EmiLiozzi, A., 1974: La collezione Rossi-Danielli, Roma.

EtTlinger, E. - Simonett, C., 1952: Römische Keramik aus dem Schutthügel von Vindonissa, Basel.

Favaretto, I., 1982: Ceramica Greca, Italiota ed 
Etrusca del Museo Provinciale di Torcello, Roma.

Felletti MAJ, G., 1957: Via Nomentana. Ministero delle Comunicazioni: nuovi rinvenimenti, Notizie Scavi, XI, 331-334.

Filtzinger, P., 1972: Die Römische Keramik aus dem Militärbereich von Novaesium, Berlin.

FORTI, L., 1962: Gli unguentari del primo periodo ellenistico, Rendiconti dell'Accademia di Archeologia, Lettere e Belle Arti di Napoli, 37, 143-155.

Fortunati ZucCala, M., 1979: Gropello Cairoli (Pavia) - La necropoli romana, Notizie Scavi, XXXIII, 5-88.

Hayes, J. W., 1976: Roman pottery in the Royal Ontario Museum - a catalogue, Toronto.

IsINGS, C., 1957: Roman glass from dated finds, Groningen - Djakarta.

Joly, E. - Garraffo, S. - Mandruzzato, A., 1992: Materiali minori dallo scavo del teatro di Leptis Magna, Quaderni di Archeologia della Libya, $15,25-233$.

LoeschKe, K., 1909: Keramik Funde in Haltern. Ein Beitrag zur Geschichte der Augusteischen Kultur, Deutschland Mitteilungen Altertum - Kommission für Westfalen, V.

MaetzKe, G., 1964: Florinas (Sassari). Necropoli a enkytrismos in località Cantaru Ena, Notizie Scavi, XVIII, 280-314.

Mercando, L., 1974: La necropoli romana di Portorecanati, Notizie Scavi, XXVIII, 145-430.

Morel, J. P., 1981: Céramique campanienne: les formes, Rome.
Moretti, A. - Sgubini Moretti, M. (a cura di), 1983: I Curunas di Tuscania, Roma.

Passi Pitcher, L., 1987: Sub Ascia. Una necropoli romana a Nave, Modena.

Plesnicar - GeC, L., 1972: Severno Emonsko grobisce. The Northern necropolis of Emona, Ljubljana.

Pulak, C. - Townsend, R. F., 1987: The Hellenistic shipwreck at Serçe Limani, Turkey: preliminary report, American Journal of Archaeology, 91.1, 31-57.

RICCI, A. (a cura di), 1985: Settefinestre. Una villa schiavistica nell'Etruria Romana 2. La villa e $i$ suoi reperti, Modena.

Santrot, M. - Santrot, J. - Querré, G. - Vaury, N., 1992: Production, importation et consommation de céramique à Bolsena au IIe S. av. J.-C. et au Ier S. ap. J.-C.: Quelques apports de la citerne 5, Rei Cretariae Romanae Fautorum Acta XXXI/XXXII, Congressus septimus decimus Rei Cretariae Romanae fautorum Ticinensis, MCMXC, in Agro Rauracense, 41-55.

Torelli, M. - PoHl, I., 1973: Veio. Scoperta di un piccolo santuario etrusco in località Campetti, Notizie Scavi, XXVII, 331-334.

Vegas, M., 1973: Cerámica común romana del Mediterráneo occidental, Barcelona.

ZAMPIERI, G., 1991: Ceramica greca, etrusca e italiota del Museo Civico di Padova, I, Roma.

Zevi, F. - Pohl, I., 1970: Ostia, saggi di scavo, Notizie Scavi, XXIV, supp. I, 7-246. 